\title{
December 2020
}

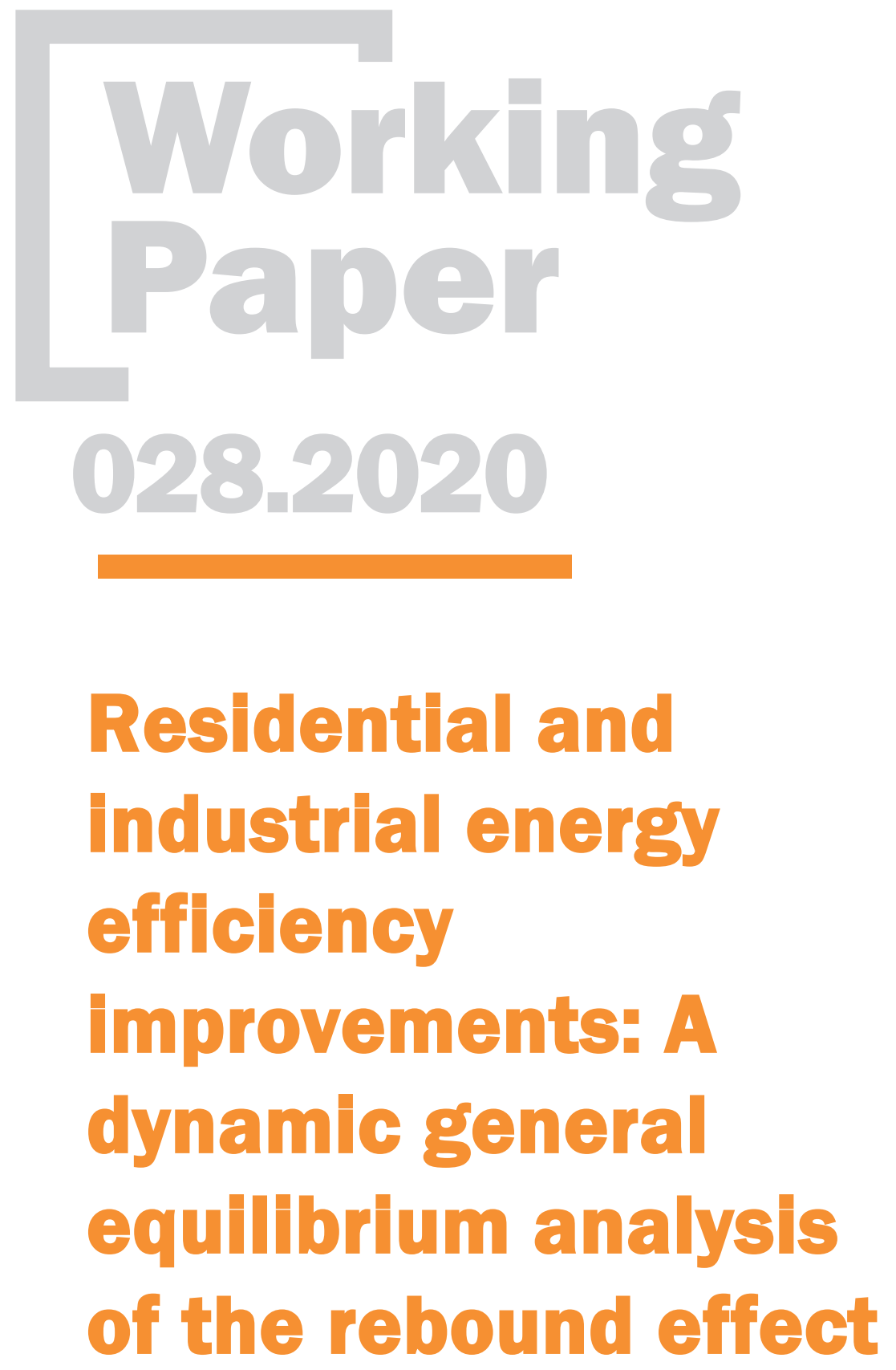

Sondes Kahouli, Xavier Pautrel 


\title{
Future Energy Program \\ Series Editor: Manfred Hafner
}

\section{Residential and industrial energy efficiency improvement: A dynamic general equilibrium analysis of the rebound effect}

\author{
By Sondes Kahouli, Université de Bretagne Occidentale \\ Xavier Pautrel, Université d'Angers
}

\begin{abstract}
Summary
The aim of this paper is to investigate bi-directional spillovers into residential and industrial sectors induced by energy efficiency improvement (EEI) in both the short- and long-term, and the impact of nesting structure as well as the size of elasticities of substitution of production and utility functions on the magnitude and the transitional dynamic of rebound effect. Developing a dynamic general equilibrium model, we demonstrate that residential EEls spillovers into the industrial sector through the labor supply channel and industrial EEls spill-overs into the residential sector through the conventional income channel. Numerical simulations calibrated on the U.S. suggest that not taking into account these spillover effects could lead to mis-estimate the rebound effect especially of residential sector EEls. We also demonstrate how the size and the duration of the rebound effect depend on the value of elasticities of substitution. Especially, the elasticity of substitution between energy and non-energy consumption in household utility and the elasticity of substitution between physical capital and labor in production play a major role. Numerical simulations suggest that alternative sets of value for the elasticities of substitution may give sizable different patterns of rebound effects in both the short- and long-term. In policy terms, our results suggest that energy effciency policies should be implemented simultaneously with rebound effect offsetting policies by considering short- and long-term wide-economy feedbacks. As a consequence, they recall for considering debates about what type of policy pathways is more effective in mitigating the rebound effect.
\end{abstract}

Keywords: Energy efficiency, Rebound effect, Transitional dynamics, Residential energy consumption, Industrial energy consumption

JEL Classification: D58, Q43

Address for correspondence:

Xavier Pautrel

Université d'Angers

GRANE; \& TEPP

13 Boulevard François Mitterrand

49000 Angers

France

E-mail: xavier.pautrel@univ-angers.fr 


\title{
Residential and industrial energy efficiency improvements: A dynamic general equilibrium analysis of the rebound effect
}

\author{
Sondès Kahouli*and Xavier PAUtrel ${ }^{\dagger}$
}

November 25, 2020

\begin{abstract}
The aim of this paper is to investigate bi-directional spillovers into residential and industrial sectors induced by energy efficiency improvement (EEI) in both the short- and long-term, and the impact of nesting structure as well as the size of elasticities of substitution of production and utility functions on the magnitude and the transitional dynamic of rebound effect.

Developing a dynamic general equilibrium model, we demonstrate that residential EEIs spillovers into the industrial sector through the labor supply channel and industrial EEIs spill-overs into the residential sector through the conventional income channel. Numerical simulations calibrated on the U.S. suggest that not taking into account these spillover effects could lead to mis-estimate the rebound effect especially of residential sector EEIs. We also demonstrate how the size and the duration of the rebound effect depend on the value of elasticities of substitution. Especially, the elasticity of substitution between energy and non-energy consumption in household utility and the elasticity of substitution between physical capital and labor in production play a major role. Numerical simulations suggest that alternative sets of value for the elasticities of substitution may give sizable different patterns of rebound effects in both the short- and long-term.

In policy terms, our results suggest that energy efficiency policies should be implemented simultaneously with rebound effect offsetting policies by considering short- and long-term wide-economy feedbacks. As a consequence, they recall for considering debates about what type of policy pathways is more effective in mitigating the rebound effect.
\end{abstract}

\section{Keywords}

Energy efficiency; Rebound effect; Transitional dynamics, Residential energy consumption; Industrial energy consumption.

\section{$J E L$ classification}

$D 58 ; Q 43$

\footnotetext{
${ }^{*}$ Université de Bretagne Occidentale. IFREMER, CNRS, UMR 6308 AMURE, IUEM. 12 rue de Kergoat, CS 93837 29238 Brest Cedex 3. France. Tel: +33(0)2 980173 81. Fax: +33(0)2 980169 35. E-mail address: sondes.kahouli@univbrest.fr.

${ }^{\dagger}$ Université d'Angers, GRANEM \& TEPP, 13 Boulevard François Mitterrand, 49000 Angers, France. E-mail address: xavier.pautrel@univ-angers.fr.
} 


\section{Introduction}

While the rebound effect due to energy efficiency improvement (EEI) has been extensively studied, empirically and theoretically, in both manufacturing and residential sector, all or almost all these works, at our best knowledge, dealt with rebound effect in each sectors separately. Nevertheless, energy efficiency improvements in one sector could spill-over into the second sector, affecting the rebound effect on sectoral energy consumption and on global energy consumption. The purpose of the paper is to fill the gap by building a theoretical model in order to investigate the channels through which energy efficiency improvements in industrial sectors could affect energy consumption in the residential sector, and vice-versa, both in the short- and long-term. It also focuses on the impact of nesting structure as well as the size of elasticities of substitution of production and utility functions on the magnitude and the transitional dynamic of rebound effect.

There is a large body of theoretical and empirical literature dealing with energy efficiency and rebound effect that are expected to happen. In general, energy efficiency refers to the amount of output that can be produced with a given input of energy. It is usually measured as the amount of energy output for a given energy input and listed as a percentage between $0 \%$ and $100 \%$.

In many cases, improvements in energy efficiency results in energy savings that are lower than expected. Sometimes, it can even induce an increase in energy consumption. This phenomenon is called the rebound effect or Jevons' paradox (Jevons, 1865). It appears when consumers do not simply replace an old product with a more efficient one which fulfills the same function, but upgrade to a larger product and often involves supplementary energy consumption that offset part of the energy savings. So far, the rebound effect has been mainly associated with energy use and the question how energy efficiency improvements affect energy consumption. Khazzoom $(1980,1987)$ and Brookes (1990) proposed a precise definition of the rebound effect, which can easily be applied to resource use in general. According to this definition, if technological progress makes equipment more energy efficient, less energy is needed to produce the same amount of product or service. However, the amount of product or service usually does not stay the same. Because the equipment becomes more energy efficient, the cost per unit of product or service that is produced with this equipment falls which, in turn, increases the demand for the product or the service. Since 1980s, several empirical studies confirm the existence of rebound effect with respect to, both, residential and firms energy demand. The economic literature recognize the existence of rebound effect but disagree about its magnitude.

The definition of the rebound effect encompasses different mechanisms that may reduce energy savings derived from the improvements in energy efficiency (Greene et al. (1999), Sorrell and Dimitropoulos (2008), Orea et al. (2015)). Through three typologies of rebound effects, this definition distinguishes a microeconomic and macroeconomic views of such phenomenon:

- Direct rebound effects. It is a pure price effect. It refers to the fact that improved energy efficiency for a particular energy servic ${ }^{2}$ will decrease the effective price of that service and should therefore lead to an increase in its consumption (Khazzoom, 1980). This will tend to offset the reduction in energy consumption provided by the efficiency improvement. For consumers, the direct effect of a price decrease may be decomposed into a substitution effect and an income effect.

- Indirect effects. The lower effective price of the energy service may lead to an increase in the demand for other goods, services. It also may lead to economic growth since it may induce an increase in factors of production that require energy for their provision. The size of the indirect effect for a consumer is dependent on the share of the consumer's total income spent on energy

\footnotetext{
${ }^{1}$ Nevertheless, other types of output can also be used. For example, one can define energy efficiency as the ratio of output of performance, service, goods or energy, to input of energy for a system. To illustrate these kinds of output, thermal comfort in a building is an example of performance, transport of persons or of information is a service, a smartphone is a good, the production of which requires energy. As for the system, it could be an individual energy equipment, a building, an industrial process, a firm, a sector or an entire economy. Improvements in energy efficiency may reduce the energy used by that system.

${ }^{2}$ Energy services refer to the commodity which is used or demanded, i.e. heating space and water, cooling space, transportation, refrigeration.
} 
services. For firms in a given sector, indirect effect result from, both, the increased demand for non-energy inputs to their production process as a result of increased demand for production, and the effect of the lower cost of one sector's output on production costs of other sectors.

- Economy wide effects. It captures the structural changes in the economy due to the variation of prices of goods and services subsequent to the improvement of energy efficiency. It means that a fall in the real price of energy services may reduce the price of intermediate and final goods throughout the economy, leading to a series of price and quantity adjustments, with energy-intensive goods and sectors likely to gain at the expense of less energy-intensive ones.

In addition to the large body of empirical literature estimating the size of the rebound effect in residential and industrial sectors $3^{3}$ the theoretical literature gives another comprehensive overview on the rebound effect. Most formal theoretical analyses of rebound effects have focused on partial equilibrium settings that hold some prices fixed (Dubin et al. (1986), Frondel et al. (2008), Greene et al. (1999), Klein (1987, 1988), Nadel (1993), Schwarz and Taylor (1995), West (2004), Greening et al. (2000), Sorrell and Dimitropoulos (2008), Sorrell et al. (2009), andVan den (2011)). These studies assume that there are no changes in prices or nominal incomes following the efficiency improvement, and that the impacts are limited to the direct market for household energy use. This approach gives a partial equilibrium figure which is generally known as the direct rebound effect. A few researches like for example Dufournaud et al. (1994) considered full general equilibrium economy-wide rebound effects from increased energy efficiency in the household sector. It examines the impacts of increasing efficiency in domestic wood stoves. Druckman et al. (2011), Freire-Gonzalez (2011) and Thomas and Thomas and Azevedo (2013a, 2013b) use a fixed price input-output model to study indirect rebound effects resulting from household income induced by energy efficiency improvements and spent on nonenergy commodities. This work considers changes in energy use in production, as well as household consumption. However, it is still studying the issue based on a partial equilibrium approach as it fails to incorporate endogenous prices, incomes or factor supply effects. As a consequence, there is a need to consider general equilibrium models to study in a more comprehensive context consequences of energy efficiency and the associated rebound effect.

Indeed, since a long time Jevons (1865) was especially worried about general equilibrium channels. More recently, his concern has been reinforced by insights from computable general equilibrium models suggesting a potential for strong rebound effects through economy-wide or alternatively indirect channels (Allan et al., 2009). In this context, many have called for theoretical research to illuminate these channels (Dimitropoulos (2007), Turner (2013), Borenstein (2015). Therefore, there has been extensive investigation of the economy-wide rebound effects resulting from energy efficiency improvements in production using a computable general equilibrium (CGE) modelling approach (Dimitropoulos (2007), Turner (2013)). However, very few studies have attempted to measure the economy-wide impacts of energy efficiency improvements in the household sector (Lecca et al., 2014). More interestingly, there is also a very few studies of rebound effect by considering interactions between production and consumption sectors. For example, Lemoine (2020) develops an analytically general equilibrium framework to study the implications of improved energy efficiency but he did not take into account feedbacks between production and consumption sectors.

This paper is aimed at filling the gap by building a general equilibrium framework where we allow for interaction between residential and industrial sectors in order to study consequences of energy efficiency improvement, in particular, the magnitude and the short- and long-run transitional dynamic of the rebound effect. We consider a variety of possible feedbacks affecting energy use in both residential (household) and production (industry) sectors. In particular, we focus on bi-directional energy spillover effects expected to spread in the residential and industrial sectors and we look at general

\footnotetext{
${ }^{3}$ The empirical literature usually focuses on the estimation of the direct rebound effect in the residential sector. However, measurements of the direct rebound effect for industrial and commercial sectors are very limited and focus on the short-run (Nadel (1993), Eto et al. (1995)).

4 Lemoine (2020)'s theoretical contribution is a decomposition of the general equilibrium response to improved efficiency. He fully endogenize all prices, the supply of labor, and the supply of energy in an economy with an arbitrary number of consumption good sectors.
} 
equilibrium channels through which these spillover operates. We quantify the rebound effect through numerical simulation after calibrating our model on the 2018 U.S. economy.

In this framework, we also investigate how important is the choice of, both, the nested structure of function, i.e. production function for firms and utility function for households, as well as the size of the elasticity of substitution between energy, labour, and physical capital in this production function and between energy and non-energy consumption in household utility function. This choice is particularly important for analyzing the magnitude and the short- and long-run transitional dynamics of rebound effect through its impact on the demand for energy and other inputs. Indeed, by studying all possible nesting structure for the constant elasticity of substitution, Van der Werf (2008) argues that the sizes of elasticities in the production function is a crucial parameter for climate policy modeling. This may also hold for energy and rebound effect modeling. For example, in Sorrell (2014) the ease of substitution between energy and other inputs and the size of the rebound effects is investigated thoroughly. By the same, Locca et al (2014) argue that a key parameter that drives rebound analysis is the elasticity of substitution between aggregate energy and non-energy goods and services in the household's utility function 5 .

Our main contributions are twofold. First, our theoretical model demonstrates that energy efficiency improvements in one sector may spillover into the other sector directly affecting its energy consumption. It also may affect the wide-economy energy consumption as well as the magnitude and the duration of the rebound effect in the sector in which the energy efficiency improvement occurred. The spillovers of EEIs in residental sector into industrial sector go through the labor supply channel and the spillovers of EEIs in industrial sector into residential sector go through the income channel. Numerical simulations calibrated on the U.S. economy show that such a spillovers affect the rebound effect in both the short- and long- term. They also show that not taking into account these spillovers effects could lead to mis-estimate the rebound effect especially for EEIs in the residential sector, in both the short- and long-term.

Our second main contribution is to demonstrate that the size and the duration of the rebound effect does not only depend on the value of elasticity of substitution between physical capital/labor and energy in the production function. Interestingly, they also depend on the value of elasticity of substitution between energy and non-energy consumption in household utility function and on the value of the elasticity of substitution between physical capital and labor. This result highlights the importance to introduce physical capital in the general equilibrium analysis of the rebound effect. Performing a comparative analysis of the short- and long- term effect of EEIs depending on whether we select values estimated by Van der Werf (2008) or values estimated by Bosetti et al. (2006) reveals sizable differences in the size and the duration of the rebound effect. This result calls for systematic evaluations of the elasticities of substitution between physical capital and labor in production and between non-energy and energy consumption in utility, in a similar way that it has be done for the elasticity of substitution between non-energy and energy inputs in production.

The paper unfolds as follows. In section 2, we present the theoretical model and we derive analytical results about the steady-state. In section 3 we compute numerical simulations (calibrated in the 2018 U.S. economy) for both the short- and long-term and investigate the role played by the elasticity of substitution between energy and physical capital/labor on one hand, and between physical capital and labor on the other hand. Finally, in Section 4 , we present conclude and give some policy implications.

\section{The model}

In this section, we present the model, we derive the intertemporal general equilibrium and study the steady-state equilibrium.

\footnotetext{
${ }^{5}$ By extension, in some modeling exercise taking into account the sector of the production of energy, it was shown that the key parameters in determining the size of rebound effects are elasticities of substitution in production of energy for other inputs as well as price elasticities of direct and derived demands for energy through trade -particularly in small open economies where energy itself is traded- (Saunders (1992), Allan et al. (2007), Hanley et al. (2009)).
} 


\section{$2.1 \quad$ Firms}

We assume that firms produce an homogenous good, denoted $y$, used to final and energy consumption in residential and producing sectors as well as to physical capital accumulation. They operate under perfect competition with a technology defined by the following nested Constant Elasticity Substitution (CES) production function:

$$
\begin{aligned}
y(t)=A\left[(1-a) Z(t)^{\frac{\sigma_{\mathrm{KL}, \mathrm{E}}-1}{\sigma_{\mathrm{LL}, \mathrm{E}}}}+a\left(\varepsilon_{f} E_{f}(t)\right)^{\frac{\sigma_{\mathrm{KL}, \mathrm{E}}-1}{\sigma_{\mathrm{KL}, \mathrm{E}}}}\right]^{\frac{\sigma_{\mathrm{KL}, \mathrm{E}}}{\sigma_{\mathrm{KL}, \mathrm{E}}-1}} \\
\text { with } \left.\quad Z(t) \equiv\left[\beta K^{\frac{\sigma_{\mathrm{K}, \mathrm{L}}-1}{\sigma_{\mathrm{K}, \mathrm{L}}}}+(1-\beta) L^{\frac{\sigma_{\mathrm{K}, \mathrm{L}}-1}{\sigma_{\mathrm{K}, \mathrm{L}}}}\right]^{\frac{\sigma_{\mathrm{K}, \mathrm{L}}}{\sigma_{\mathrm{K}, \mathrm{L}}-1}}, \quad(a, \beta) \in\right] 0,1[,
\end{aligned}
$$

where $K$ denotes the aggregate stock of physical capital, $L$ the labor supply, $E_{f}$ the energy consumption in production and $\varepsilon_{f}$ its efficiency.

We note $\sigma_{\mathrm{KL}, \mathrm{E}} \geq 0$ the elasticity of substitution between capital/labor and energy, and $\sigma_{\mathrm{K}, \mathrm{L}} \geq 0$ is the elasticity of substitution between capital and labor.

Firms maximize their profits $y(t)-r(t) K(t)-w(t) L(t)-p_{f} E_{f}(t)$, where $p_{f}$ is the price of energy in production, leading to the first order conditions:

$$
\begin{aligned}
& r=A\left[(1-a) Z^{\frac{\sigma_{\mathrm{KL}, \mathrm{E}}-1}{\sigma_{\mathrm{KL}, \mathrm{E}}}}+a\left(\varepsilon_{f} E_{f}\right)^{\frac{\sigma_{\mathrm{KL}, \mathrm{E}}-1}{\sigma_{\mathrm{KL}, \mathrm{E}}}}\right]^{\frac{1}{\sigma_{\mathrm{KL}, \mathrm{E}}-1}}(1-a) Z^{\frac{\sigma_{\mathrm{KL}, \mathrm{E}}-1}{\sigma_{\mathrm{KL}, \mathrm{E}}}} \frac{\beta K^{\frac{-1}{\sigma_{\mathrm{K}, \mathrm{L}}}}}{\beta K^{\frac{\sigma_{\mathrm{K}, \mathrm{L}}-1}{\sigma_{\mathrm{K}, \mathrm{L}}}}+(1-\beta) L^{\frac{\sigma_{\mathrm{K}, \mathrm{L}}-1}{\sigma_{\mathrm{K}, \mathrm{L}}}}} \\
& p_{f}=A\left[(1-a) Z^{\frac{\sigma_{\mathrm{KL}, \mathrm{E}}-1}{\sigma_{\mathrm{KL}, \mathrm{E}}}}+a\left(\varepsilon_{f} E_{f}\right)^{\frac{\sigma_{\mathrm{KL}, \mathrm{E}}-1}{\sigma_{\mathrm{KL}, \mathrm{E}}}}\right]^{\frac{1}{\sigma_{\mathrm{KL}, \mathrm{E}}-1}} a \varepsilon_{f}^{\frac{\sigma_{\mathrm{KL}, \mathrm{E}}-1}{\sigma_{\mathrm{KL}, \mathrm{E}}}} E_{f}^{\frac{1}{\sigma_{\mathrm{KL}, \mathrm{E}}-1}} \\
& w=A\left[(1-a) Z^{\frac{\sigma_{\mathrm{KL}, \mathrm{E}}-1}{\sigma_{\mathrm{KL}, \mathrm{E}}}}+a\left(\varepsilon_{f} E_{f}\right)^{\frac{\sigma_{\mathrm{KL}, \mathrm{E}}-1}{\sigma_{\mathrm{KL}, \mathrm{E}}}}\right]^{\frac{1}{\sigma_{\mathrm{KL}, \mathrm{E}-1}}}(1-a) Z^{\frac{\sigma_{\mathrm{KL}, \mathrm{E}}-1}{\sigma_{\mathrm{KL}, \mathrm{E}}}} \frac{(1-\beta) L^{\frac{-1}{\sigma_{\mathrm{K}, \mathrm{L}}}}}{\beta K^{\frac{\sigma_{\mathrm{K}, \mathrm{L}}-1}{\sigma_{\mathrm{K}, \mathrm{L}}}}+(1-\beta) L^{\frac{\sigma_{\mathrm{K}, \mathrm{L}}-1}{\sigma_{\mathrm{K}, \mathrm{L}}}}}
\end{aligned}
$$

Using equation (3) we obtain:

$$
\frac{Z}{\varepsilon_{f} E_{f}}=\Omega\left(\varepsilon_{f}, p_{f} ; \sigma_{\mathrm{KL}, \mathrm{E}}\right) \equiv\left(\frac{\left(\frac{p_{f}}{a A \varepsilon_{f}}\right)^{\sigma_{\mathrm{KL}, \mathrm{E}}-1}-a}{1-a}\right)^{\frac{\sigma_{\mathrm{KL}, \mathrm{E}}}{\sigma_{\mathrm{LL}, \mathrm{E}}-1}}>0
$$

Lemma 1. $\partial \Omega\left(\varepsilon_{f}, p_{f} ; \sigma_{\mathrm{KL}, \mathrm{E}}\right) / \partial \varepsilon_{f}<0$ and $\partial \Omega\left(\varepsilon_{f}, p_{f} ; \sigma_{\mathrm{KL}, \mathrm{E}}\right) / \partial p_{f}>0, \forall \sigma_{\mathrm{KL}, \mathrm{E}}$.

Proof. From equation (5).

Furthermore, equations (2) and (4) give the ratio physical capital to labor $K / L$ :

$$
\left(\frac{K}{L}\right)^{1 / \sigma_{\mathrm{K}, \mathrm{L}}}=\left(\frac{\beta}{1-\beta}\right) \frac{w}{r}
$$

Using equations (3) and (2), it comes:

$$
r=\left(\frac{1-a}{a}\right) p_{f} \Omega\left(\varepsilon_{f}, p_{f} ; \sigma_{\mathrm{KL}, \mathrm{E}}\right)^{\frac{\sigma_{\mathrm{KL}, \mathrm{E}}-1}{\sigma_{\mathrm{KL}, \mathrm{E}}}} \frac{\beta E_{f} K^{\frac{-1}{\sigma_{\mathrm{K}, \mathrm{L}}}}}{\beta K^{\frac{\sigma_{\mathrm{K}, \mathrm{L}}-1}{\sigma_{\mathrm{K}, \mathrm{L}}}}+(1-\beta) L^{-\frac{\sigma_{\mathrm{K}, \mathrm{L}}-1}{\sigma_{\mathrm{K}, \mathrm{L}}}}}
$$

Equation (3) enables us to rewrite equation (1) as:

$$
y=\frac{p_{f} E_{f}}{a}\left[(1-a) \Omega\left(\varepsilon_{f}, p_{f} ; \sigma_{\mathrm{KL}, \mathrm{E}}\right)^{\frac{\sigma_{\mathrm{KL}, \mathrm{E}}-1}{\sigma_{\mathrm{KL}, \mathrm{E}}}}+a\right]=\left(\frac{p_{f}}{a}\right)^{\sigma_{\mathrm{KL}, \mathrm{E}}}\left(A \varepsilon_{f}\right)^{1-\sigma_{\mathrm{KL}, \mathrm{E}}} E_{f}
$$

From equations (3) and (4) we have:

$$
w=\mathcal{A}\left(\varepsilon_{f}, p_{f} ; \sigma_{\mathrm{KL}, \mathrm{E}}\right)(1-\beta)\left[\beta K^{\frac{\sigma_{\mathrm{K}, \mathrm{L}}-1}{\sigma_{\mathrm{K}, \mathrm{L}}}}+(1-\beta) L^{\frac{\sigma_{\mathrm{K}, \mathrm{L}}-1}{\sigma_{\mathrm{K}, \mathrm{L}}}}\right]^{\frac{1}{\sigma_{\mathrm{K}, \mathrm{L}}-1}} L^{\frac{-1}{\sigma_{\mathrm{K}, \mathrm{L}}}}
$$

where $\mathcal{A}\left(\varepsilon_{f}, p_{f} ; \sigma_{\mathrm{KL}, \mathrm{E}}\right) \equiv \frac{(1-a) p_{f}}{a \varepsilon_{f}} \Omega\left(\varepsilon_{f}, p_{f} ; \sigma_{\mathrm{KL}, \mathrm{E}}\right)^{\frac{-1}{\sigma_{\mathrm{KL}, \mathrm{E}}}}$. 
Lemma 2. We have $\partial \mathcal{A}\left(\varepsilon_{f}, p_{f} ; \sigma_{\mathrm{KL}, \mathrm{E}}\right) / \partial \varepsilon_{f}>0$ and $\partial \mathcal{A}\left(\varepsilon_{f}, p_{f} ; \sigma_{\mathrm{KL}, \mathrm{E}}\right) / \partial p_{f}<0, \forall \sigma_{\mathrm{KL}, \mathrm{E}}$.

Proof. From Lemma 1.

Then, equations (6) and (9) give:

$$
r=\mathcal{A}\left(\varepsilon_{f}, p_{f} ; \sigma_{\mathrm{KL}, \mathrm{E}}\right) \beta\left[\beta K^{\frac{\sigma_{\mathrm{K}, \mathrm{L}}-1}{\sigma_{\mathrm{K}, \mathrm{L}}}}+(1-\beta) L^{\frac{\sigma_{\mathrm{K}, \mathrm{L}}-1}{\sigma_{\mathrm{K}, \mathrm{L}}}}\right]^{\frac{1}{\sigma_{\mathrm{K}, \mathrm{L}}-1}} K^{\frac{-1}{\sigma_{\mathrm{K}, \mathrm{L}}}}
$$

Using equations (10) and (7) gives:

$$
E_{f}=\frac{\left[\beta K^{\frac{\sigma_{\mathrm{K}, \mathrm{L}}-1}{\sigma_{\mathrm{K}, \mathrm{L}}}}+(1-\beta) L^{\frac{\sigma_{\mathrm{K}, \mathrm{L}}-1}{\sigma_{\mathrm{K}, \mathrm{L}}}}\right]^{\frac{\sigma_{\mathrm{K}, \mathrm{L}}}{\sigma_{\mathrm{K}, \mathrm{L}}-1}}}{\varepsilon_{f} \Omega\left(\varepsilon_{f}, p_{f} ; \sigma_{\mathrm{KL}, \mathrm{E}}\right)}
$$

\subsection{Households}

There is a representative household that is infinitely lived, with preferences given by:

$$
\int_{t=0}^{\infty} e^{-\rho t} U(\bar{C}(t), \ell(t)) d t
$$

where $\ell(t) \in] 0,1[$ is the fraction of his unitary time endowment devoted to leisure and $\bar{C}(t)$ is aggregate consumption. $\rho>0$ is the time discount rate and $U$ is the period utility function. Aggregate consumption is defined as:

$$
\left.\bar{C}(t)=\left[(1-b) c(t)^{\frac{\sigma_{\mathrm{C}, \mathrm{E}}-1}{\sigma_{\mathrm{C}, \mathrm{E}}}}+b\left(\varepsilon_{r} E_{r}(t)\right)^{\frac{\sigma_{\mathrm{C}, \mathrm{E}}-1}{\sigma_{\mathrm{C}, \mathrm{E}}}}\right]^{\frac{\sigma_{\mathrm{C}, \mathrm{E}}}{\sigma_{\mathrm{C}, \mathrm{E}}-1}} \quad \text { with } \quad b \in\right] 0,1[
$$

where $c(t)$ is the household consumption in final goods and $E_{r}(t)$ is the residential consumption of energy whose efficiency is captured by the elasticity $\varepsilon_{r}$. As for $\sigma_{\mathrm{C}, \mathrm{E}} \geq 0$, it represents the elasticity of substitution between final goods and energy consumptions.

The representative household owns physical capital that he rents to firms. He supplies $L=1-\ell$ of his time endowment as labor. His budget constraint is:

$$
\dot{K}(t)=r(t) K(t)+w(t)(1-\ell(t))-c(t)-p_{r} E_{r}(t)
$$

where $p_{r}>0$ denotes the unitary price of residential energy services.

Household's choices are represented by the following consumer's problem:

$$
\max _{c, \ell, K, E_{r}} \int_{t=0}^{\infty} e^{-\rho t} U(\bar{C}(t), \ell(t)) d t \quad \text { s.t. }\left\{\begin{array}{l}
\dot{K}(t)=r(t) K(t)-w(t)(1-\ell(t))-c(t)-p_{r} E_{r}(t) \\
\bar{C}(t)=\left[(1-b) c(t)^{\frac{\sigma_{C, \mathrm{E}}-1}{\sigma_{\mathrm{C}, \mathrm{E}}}}+b\left(\varepsilon_{r} E_{r}(t)\right)^{\frac{\sigma_{\mathrm{C}, \mathrm{E}}-1}{\sigma_{\mathrm{C}, \mathrm{E}}}}\right]^{\frac{\sigma_{\mathrm{C}, \mathrm{E}}}{\sigma_{\mathrm{C}, \mathrm{E}}-1}}
\end{array}\right.
$$

Lagrangian is written as:

$$
\mathcal{H}=U(\bar{C}, \ell)+\mu_{k}\left[r K-w(1-\ell)-c-p_{r} E_{r}\right]
$$

The first-order conditions are:

$$
\begin{array}{lll}
\frac{\partial \mathcal{H}}{\partial c}=0 & \Rightarrow & U_{\bar{C}} \bar{C}_{c}-\mu_{k}=0 \\
\frac{\partial \mathcal{H}}{\partial e_{r}}=0 & \Rightarrow & U_{\bar{C}} \bar{C}_{E_{r}}-\mu_{k} p_{r}=0 \\
\frac{\partial \mathcal{H}}{\partial \ell}=0 \quad \Rightarrow & U_{\ell}-\mu_{k} w=0 \\
\frac{\partial \mathcal{H}}{\partial K}=-\dot{\mu_{k}}+\rho \mu_{k} & \Rightarrow & r \mu_{k}=-\dot{\mu_{k}}+\rho \mu_{k}
\end{array}
$$

Equations (17) and (18) give:

$$
E_{r}=\mathcal{S}\left(\varepsilon_{r}, p_{r} ; \sigma_{\mathrm{C}, \mathrm{E}}\right) c \quad \text { with } \quad \mathcal{S}\left(\varepsilon_{r}, p_{r} ; \sigma_{\mathrm{C}, \mathrm{E}}\right) \equiv\left(\frac{b}{1-b}\right)^{\sigma_{\mathrm{C}, \mathrm{E}}} p_{r}^{-\sigma_{\mathrm{C}, \mathrm{E}}} \varepsilon_{r}^{\sigma_{\mathrm{C}, \mathrm{E}}-1}
$$


Lemma 3. We have $\partial \mathcal{S}\left(\varepsilon_{r}, p_{r} ; \sigma_{\mathrm{C}, \mathrm{E}}\right) / \partial \varepsilon_{r} \geq 0$ when $\sigma_{\mathrm{C}, \mathrm{E}} \geq 1$ and $\partial \mathcal{S}\left(\varepsilon_{r}, p_{r} ; \sigma_{\mathrm{C}, \mathrm{E}}\right) / \partial p_{r}<0 \forall \sigma_{\mathrm{C}, \mathrm{E}}>0$. Proof. From equation (21).

As a consequence, utility given by 13 can be written as:

$$
\bar{C}=\mathcal{U}\left(\varepsilon_{r}, p_{r} ; \sigma_{\mathrm{C}, \mathrm{E}}\right) c \quad \text { with } \quad \mathcal{U}\left(\varepsilon_{r}, p_{r} ; \sigma_{\mathrm{C}, \mathrm{E}}\right) \equiv\left[(1-b)+b\left(\varepsilon_{r} \mathcal{S}\left(\varepsilon_{r}, p_{r} ; \sigma_{\mathrm{C}, \mathrm{E}}\right)\right)^{\frac{\sigma_{\mathrm{C}, \mathrm{E}}-1}{\sigma_{\mathrm{C}, \mathrm{E}}}}\right]^{\frac{\sigma_{\mathrm{C}, \mathrm{E}}}{\sigma_{\mathrm{C}, \mathrm{E}}-1}}
$$

Furthermore, leisure is expressed as a function of consumption and wage:

$$
U_{\ell}=w U_{\bar{C}} \bar{C}_{c}
$$

and the law of motion of consumption is given by

$$
U_{c c} \dot{c}=(r-\rho) U_{c}-U_{c, \ell} \dot{\ell}
$$

\subsection{Intertemporal general equilibrium}

The aggregate resource constraint is:

$$
y=c+\dot{K}+p_{r} E_{r}+p_{f} E_{f}
$$

Equilibrium in labor market is described by:

$$
L=1-\ell
$$

and the aggregate energy use $E$ is defined as:

$$
E=E_{f}+E_{r}
$$

Assuming for simplicity that:

$$
U(\bar{C}, \ell) \equiv \log \bar{C}+\varphi \log \ell
$$

then from equation $(23)$, we obtain:

$$
\ell=\varphi \frac{c}{w}
$$

Using equations (25), (8), (11) and (29), we obtain the law of motion of $K$ :

$$
\begin{aligned}
\dot{K}=\mathcal{A}\left(\varepsilon_{f}, p_{f} ; \sigma_{\mathrm{KL}, \mathrm{E}}\right)\left[\beta K^{\frac{\sigma_{\mathrm{K}, \mathrm{L}}-1}{\sigma_{\mathrm{K}, \mathrm{L}}}}+(1-\beta)\right. & \left.(1-\ell)^{\frac{\sigma_{\mathrm{K}, \mathrm{L}}-1}{\sigma_{\mathrm{K}, \mathrm{L}}}}\right]^{\frac{\sigma_{\mathrm{K}, \mathrm{L}}}{\sigma_{\mathrm{L}, \mathrm{L}}-1}} \\
\times & \left\{1-\frac{\left[1+p_{r} \mathcal{S}\left(\varepsilon_{r}, p_{r} ; \sigma_{\mathrm{C}, \mathrm{E}}\right)\right](1-\beta)(1-\ell)^{\frac{-1}{\sigma_{\mathrm{K}, \mathrm{L}}}} \ell}{\varphi\left[\beta K^{\frac{\sigma_{\mathrm{K}, \mathrm{L}}-1}{\sigma_{\mathrm{K}, \mathrm{L}}}}+(1-\beta)(1-\ell)^{\frac{\sigma_{\mathrm{K}, \mathrm{L}}-1}{\sigma_{\mathrm{K}, \mathrm{L}}}}\right]}\right\}
\end{aligned}
$$

Using equation (10), equation 24 gives:

$$
\dot{c}=\left(\mathcal{A}\left(\varepsilon_{f}, p_{f} ; \sigma_{\mathrm{KL}, \mathrm{E}}\right)\left[\beta K^{\frac{\sigma_{\mathrm{K}, \mathrm{L}}-1}{\sigma_{\mathrm{K}, \mathrm{L}}}}+(1-\beta)(1-\ell)^{\frac{\sigma_{\mathrm{K}, \mathrm{L}}-1}{\sigma_{\mathrm{K}, \mathrm{L}}}}\right]^{\frac{1}{\sigma_{\mathrm{K}, \mathrm{L}}-1}} \beta K^{\frac{-1}{\sigma_{\mathrm{K}, \mathrm{L}}}}-\rho\right) c
$$

Differentiating equation (29) with respect to time and using equation (9), the law of motion of the portion of time endowed in production is:

$$
\begin{aligned}
& \dot{\ell}=\left[1+\left(\frac{\sigma_{\mathrm{K}, \mathrm{L}}^{-1} \beta K^{\frac{\sigma_{\mathrm{K}, \mathrm{L}}-1}{\sigma_{\mathrm{K}, \mathrm{L}}}}}{\beta K^{\frac{\sigma_{\mathrm{K}, \mathrm{L}}-1}{\sigma_{\mathrm{L}, \mathrm{L}}}}+(1-\beta)(1-\ell)^{\frac{\sigma_{\mathrm{K}, \mathrm{L}}-1}{\sigma_{\mathrm{K}, \mathrm{L}}}}}\right) \frac{\ell}{1-\ell}\right]^{-1} \times \\
& {\left[\beta \mathcal{A}\left(\varepsilon_{f}, p_{f} ; \sigma_{\mathrm{KL}, \mathrm{E}}\right)\left[\beta K^{\frac{\sigma_{\mathrm{K}, \mathrm{L}}-1}{\sigma_{\mathrm{K}, \mathrm{L}}}}+(1-\beta)(1-\ell)^{\frac{\sigma_{\mathrm{K}, \mathrm{L}}-1}{\sigma_{\mathrm{K}, \mathrm{L}}}}\right]^{\frac{1}{\sigma_{\mathrm{K}, \mathrm{L}}-1}} K^{\frac{-1}{\sigma_{\mathrm{K}, \mathrm{L}}} \times}\right.} \\
& \left.\qquad\left\{\frac{\sigma_{\mathrm{K}, \mathrm{L}}-1}{\sigma_{\mathrm{K}, \mathrm{L}}}+\left(\frac{1}{\sigma_{\mathrm{K}, \mathrm{L}}}\right) \frac{\left[1+p_{r} \mathcal{S}\left(\varepsilon_{r}, p_{r} ; \sigma_{\mathrm{C}, \mathrm{E}}\right)\right](1-\beta)(1-\ell)^{\frac{-1}{\sigma_{\mathrm{K}, \mathrm{L}}}} \ell}{\varphi\left[\beta K^{\frac{\sigma_{K, \mathrm{~L}}-1}{\sigma_{\mathrm{K}, \mathrm{L}}}}+(1-\beta)(1-\ell)^{\frac{\sigma_{\mathrm{K}, \mathrm{L}}-1}{\sigma_{\mathrm{K}, \mathrm{L}}}}\right]}\right\}\right]
\end{aligned}
$$




\subsection{Steady-state analysis}

At the steady-state, $K=K^{\star}$ and $\ell=\ell^{\star}$ are constant. As a result, from equation $(32), \dot{\ell}=0$ gives:

$$
K^{\star} \frac{-1}{\sigma_{K, \mathrm{~L}}}\left[\beta K^{\star \frac{\sigma_{\mathrm{K}, \mathrm{L}}-1}{\sigma_{\mathrm{K}, \mathrm{L}}}}+(1-\beta)\left(1-\ell^{\star}\right)^{\frac{\sigma_{\mathrm{K}, \mathrm{L}}-1}{\sigma_{\mathrm{K}, \mathrm{L}}}}\right]^{\frac{1}{\sigma_{\mathrm{K}, \mathrm{L}}-1}}=\frac{\rho}{\beta \mathcal{A}\left(\varepsilon_{f}, p_{f} ; \sigma_{\mathrm{KL}, \mathrm{E}}\right)}
$$

which defines the physical capital-labor ratio as a function of the energy-efficiency $\varepsilon_{f}$ :

$$
\frac{K^{\star}}{1-\ell^{\star}}=\kappa\left(\varepsilon_{f}, p_{f} ; \sigma_{\mathrm{KL}, \mathrm{E}}\right) \equiv\left[\frac{\left(\frac{\rho}{\beta \mathcal{A}\left(\varepsilon_{f}, p_{f} ; \sigma_{\mathrm{KL}, \mathrm{E}}\right)}\right)^{\sigma_{\mathrm{K}, \mathrm{L}}-1}-\beta}{1-\beta}\right]^{-\frac{\sigma_{\mathrm{K}, \mathrm{L}}}{\sigma_{\mathrm{K}, \mathrm{L}}-1}}
$$

Lemma 4. We have $\partial \kappa\left(\varepsilon_{f}, p_{f} ; \sigma_{\mathrm{KL}, \mathrm{E}}\right) / \partial \varepsilon_{f}>0$ and $\partial \kappa\left(\varepsilon_{f}, p_{f} ; \sigma_{\mathrm{KL}, \mathrm{E}}\right) / \partial p_{f}<0, \forall\left(\sigma_{\mathrm{KL}, \mathrm{E}}, \sigma_{\mathrm{K}, \mathrm{L}}\right)$.

Proof. From equation (33) and Lemma 2 .

From equation (30) and (34), $\dot{K}=0$ gives:

$$
\ell^{\star}=\left[1+\frac{\left[1+p_{r} \mathcal{S}\left(\varepsilon_{r}, p_{r} ; \sigma_{\mathrm{C}, \mathrm{E}}\right)\right](1-\beta)}{\varphi\left[\beta \kappa\left(\varepsilon_{f}, p_{f} ; \sigma_{\mathrm{KL}, \mathrm{E}}\right)^{\frac{\sigma_{\mathrm{K}, \mathrm{L}}-1}{\sigma_{\mathrm{K}, \mathrm{L}}}}+(1-\beta)\right]}\right]^{-1}
$$

Using equation (11) and (35), we obtain:

$$
E_{f}^{\star}=\frac{\left[\beta \kappa\left(\varepsilon_{f}, p_{f} ; \sigma_{\mathrm{KL}, \mathrm{E}}\right)^{\frac{\sigma_{\mathrm{K}, \mathrm{L}}-1}{\sigma_{\mathrm{K}, \mathrm{L}}}}+1-\beta\right]^{\frac{1}{\sigma_{\mathrm{K}, \mathrm{L}}-1}}}{\varepsilon_{f} \Omega\left(\varepsilon_{f}, p_{f} ; \sigma_{\mathrm{KL}, \mathrm{E}}\right)}\left[\frac{1+p_{r} \mathcal{S}\left(\varepsilon_{r}, p_{r} ; \sigma_{\mathrm{C}, \mathrm{E}}\right)}{\beta \kappa\left(\varepsilon_{f}, p_{f} ; \sigma_{\mathrm{KL}, \mathrm{E}}\right)^{\frac{\sigma_{\mathrm{K}, \mathrm{L}}-1}{\sigma_{\mathrm{K}, \mathrm{L}}}}+1-\beta}+\frac{\varphi}{1-\beta}\right]^{-1}
$$

Proposition 1. At the steady-state equilibrium, the consumption of energy in the manufacturing sector can be expressed as:

$$
E_{f}^{\star}=\mathscr{E}_{f}\left(\varepsilon_{f}, \varepsilon_{r} ; p_{r}, p_{f}\right)
$$

1. If $\varphi=0, \partial \mathscr{E}_{f}(\cdot) / \partial \varepsilon_{r}=0$ and $\partial \mathscr{E}_{f}(\cdot) / \partial \varepsilon_{f}>0$ when $\sigma_{\mathrm{K}, \mathrm{L}}>1$.

2. If $\varphi>0, \partial \mathscr{E}_{f}(\cdot) / \partial \varepsilon_{r} \geq 0$ when $\sigma_{\mathrm{C}, \mathrm{E}} \geq 1$ and $\partial \mathscr{E}_{f}(\cdot) / \partial \varepsilon_{f}>0$ when $\sigma_{\mathrm{K}, \mathrm{L}}>1$

Proof. From equation (36), Lemma 1, and Lemma 4 .

Proposition 1 states that, energy efficiency improvements in the residential sector may spillover in industrial sector, when labor is endogenous. Then, if the elasticity of substitution between non-energy consumption and energy consumption in utility function is higher than unity, i.e. $\sigma_{\mathrm{C}, \mathrm{E}}>1$, energy efficiency in the residential sector affects positively energy consumption in the industrial sector. Indeed, industrial energy consumption directly relies on production function (equation (11)), thus, on labor -one to one- which is inversely related to leisure time (equation (35)). When $\sigma_{\mathrm{C}, \mathrm{E}}>1$, the increase in residential energy efficiency, i.e. $\varepsilon_{r}$, increases the ratio between residential energy consumption and non-energy consumption in utility (equation (21)). As a consequence, consumption and leisure time diminish, everything being equal, leading to a rise of labor supply which increases production and, therefore, energy consumption in the industrial sector, i.e. $E_{f}$.

From equations (6) and (34), it comes:

$$
w^{\star}=\left(\frac{1-\beta}{\beta}\right) \rho \kappa\left(\varepsilon_{f}, p_{f} ; \sigma_{\mathrm{KL}, \mathrm{E}}\right)^{\frac{1}{\sigma_{\mathrm{K}, \mathrm{L}}}}
$$

Then, using equations (29) and (35) we obtain:

$$
c^{\star}=\rho \kappa\left(\varepsilon_{f}, p_{f} ; \sigma_{\mathrm{KL}, \mathrm{E}}\right)^{\frac{1}{\sigma_{K, \mathrm{~L}}}}\left[\frac{\beta\left(1+p_{r} \mathcal{S}\left(\varepsilon_{r}, p_{r} ; \sigma_{\mathrm{C}, \mathrm{E}}\right)\right)}{\beta \kappa\left(\varepsilon_{f}, p_{f} ; \sigma_{\mathrm{KL}, \mathrm{E}}\right)^{\frac{\sigma_{\mathrm{K}, \mathrm{L}}-1}{\sigma_{\mathrm{K}, \mathrm{L}}}}+1-\beta}+\frac{\varphi \beta}{1-\beta}\right]^{-1}
$$


Lemma 5. At the steady-state equilibrium, the aggregate consumption is such that:

$$
c^{\star}=\mathscr{C}\left(\varepsilon_{r}, \varepsilon_{f}\right)
$$

with $\partial \mathscr{C}(\cdot) / \partial \varepsilon_{r} \geq 0$ if $\sigma_{\mathrm{C}, \mathrm{E}} \leq 1$ and, $\partial \mathscr{C}(\cdot) / \partial \varepsilon_{f}>0, \forall \sigma_{\mathrm{K}, \mathrm{L}}$.

Proof. From equations (21), (38) and Lemma 4

Using equations (21) and (38), we obtain the expression of the residential consumption of energy at the steady-state:

$$
E_{r}^{\star}=\kappa\left(\varepsilon_{f}, p_{f} ; \sigma_{\mathrm{KL}, \mathrm{E}}\right)^{\frac{1}{\sigma_{\mathrm{K}, \mathrm{L}}}} \mathcal{S}\left(\varepsilon_{r}, p_{r} ; \sigma_{\mathrm{C}, \mathrm{E}}\right) \frac{\rho}{\beta}\left[\frac{1+p_{r} \mathcal{S}\left(\varepsilon_{r}, p_{r} ; \sigma_{\mathrm{C}, \mathrm{E}}\right)}{\beta \kappa\left(\varepsilon_{f}, p_{f} ; \sigma_{\mathrm{KL}, \mathrm{E}}\right)^{\frac{\sigma_{\mathrm{K}, \mathrm{L}}-1}{\sigma_{\mathrm{K}, \mathrm{L}}}}+1-\beta}+\frac{\varphi}{1-\beta}\right]^{-1}
$$

Proposition 2. At the steady-state equilibrium, the consumption of energy in the residential sector can be expressed as:

$$
E_{r}^{\star}=\mathscr{E}_{r}\left(\varepsilon_{f}, \varepsilon_{r} ; p_{r}, p_{f}\right)
$$

with $\partial \mathscr{E}_{r}(\cdot) / \partial \varepsilon_{f}>0$ when $\sigma_{\mathrm{K}, \mathrm{L}} \geq 1$ and $\partial \mathscr{E}_{r}(\cdot) / \partial \varepsilon_{r} \geq 0$ if $\sigma_{\mathrm{C}, \mathrm{E}} \geq 1$

Proof. From equation 39 .

Proposition 2 states that energy efficiency improvements in industrial sector may spillover in residential sector: when the elasticity of substitution between physical capital and labor in production is higher than unity, i.e. $\sigma_{K, L}>1$, the energy efficiency improvement in industrial sector increases residential energy consumption. Indeed, the increase in industrial energy efficiency rises non-energy consumption (from Lemma 5), therefore, residential energy consumption, i.e. $E_{r}$ which is related to non-energy consumption (equation (21)). Interestingly, these spillover effects depend on the shape of the production function.

Finally, global energy consumption at the steady-state is:

$$
\begin{aligned}
& E^{\star}=\left[\frac{1+p_{r} \mathcal{S}\left(\varepsilon_{r}, p_{r} ; \sigma_{\mathrm{C}, \mathrm{E}}\right)}{\beta \kappa\left(\varepsilon_{f}, p_{f} ; \sigma_{\mathrm{KL}, \mathrm{E}}\right)^{\frac{\sigma_{\mathrm{K}, \mathrm{L}}-1}{\sigma_{\mathrm{K}, \mathrm{L}}}}+1-\beta}+\frac{\varphi}{1-\beta}\right]^{-1} \times \\
& \left\{\kappa\left(\varepsilon_{f}, p_{f} ; \sigma_{\mathrm{KL}, \mathrm{E}}\right)^{\frac{1}{\sigma_{\mathrm{K}, \mathrm{L}}}} \mathcal{S}\left(\varepsilon_{r}, p_{r} ; \sigma_{\mathrm{C}, \mathrm{E}}\right) \frac{\rho}{\beta}+\frac{\left[\beta \kappa\left(\varepsilon_{f}, p_{f} ; \sigma_{\mathrm{KL}, \mathrm{E}}\right)^{\frac{\sigma_{\mathrm{K}, \mathrm{L}}-1}{\sigma_{\mathrm{K}, \mathrm{L}}}}+1-\beta\right]^{\frac{1}{\sigma_{\mathrm{K}, \mathrm{L}}-1}}}{\varepsilon_{f} \Omega\left(\varepsilon_{f}, p_{f} ; \sigma_{\mathrm{KL}, \mathrm{E}}\right)}\right\}
\end{aligned}
$$

Proposition 3. At the steady-state equilibrium, the global consumption of energy in the economy can be expressed as:

$$
E^{\star}=\mathscr{E}\left(\varepsilon_{f}, \varepsilon_{r} ; p_{r}, p_{f}\right)
$$

with $\partial \mathscr{E}(\cdot) / \partial \varepsilon_{r} \geq 0$ if $\sigma_{\mathrm{C}, \mathrm{E}} \geq 1$ and $\partial \mathscr{E}(\cdot) / \partial \varepsilon_{f}>0$ if $\sigma_{\mathrm{K}, \mathrm{L}}>1$.

Proof. From equation (40) and Propositions 1 and 2.

\section{Numerical simulations}

In this section, we present numerical simulations of our theoretical model that we calibrate on the 2018 U.S. economy. In sub-section 3.1, we explain calibration of preference, technology, and energy parameters. In sub-section 3.2 , we discuss results. 


\subsection{Calibration}

Preference parameters: Following Turnovsky and Garcia-Penalosa (2008), we set the elasticity of leisure in utility to 1.75 which leads to a steady-state leisure time close to two-thirds of individual time as in Prescott (2004). The value of the elasticity of substitution between energy and non-energy consumption in utility comes from Lemoine (2020) and is very close to the value reported by de Miguel and Manzano (2011). The time discount rate $\rho$ is chosen to obtain an annual interest rate equal to $4 \%$ as usual in the literature. Finally, we calibrate energy consumption share in utility $b$ in order to match the 2018 personal consumption expenditures net of energy expenditures to residential energy consumption ratio in the U.S. economy. Personal consumption expenditures come from the FRED Economic dataset of the Saint-Louis Federal Reserve and residential energy consumption are extracted from the U.S. Energy Information Administration (EIA) State Energy Data set.

Technology parameters: We follow the Real Business Cycle literature to set the share of physical capital in production $a$ at $1 / 3$. As for elasticities of substitution in the production function, we consider the elasticity of substitution between energy $E_{f}$ and non-energy factors $Z$ as well as between physical capital and labor. The question of what values to attribute to these elasticities is the subject of recurrent debate in the current state of empirical literature. As a consequence, in our model, we use as benchmark values the estimations made by Van der Werf (2008) for the U.S. and we will also investigate alternative values estimated by other authors. Otherwise, we approximate the share of energy in industrial production $a$ by the ratio energy expenditures in the industrial sector to GDP, using the data from the U.S. EIA State Energy Data set. Finally, we calibrate the scale parameter $A$ in the production function to match the 2018 ratio of residential energy consumption estimates to the industrial energy consumption estimates as published in the U.S. EIA State Energy Data set.

Energy parameters: Values of energy efficiencies are extracted from the 2016 American Council for an Energy-Efficient Economy (ACEEE) report. As for energy prices, they come from the 2018 U.S. EIA State Energy Data set.

In Table 1, we summarize benchmark parameter values and their sources.

Table 1: Benchmark parameter values

\begin{tabular}{|c|c|c|c|}
\hline Parameter & & Value & Source \\
\hline \multicolumn{4}{|l|}{ Preference } \\
\hline - Elasticity of leisure in utility & $\varphi$ & 1.75 & Turnovsky and Garcia-Penalosa $(2008)$ \\
\hline - Elasticity of substitution between energy and non-energy consumption & $\sigma_{\mathrm{C}, \mathrm{E}}$ & 0.9 & Lemoine $(2020)$ \\
\hline - Subjective rate of time preference & $\rho$ & 0.04 & Corresponds to a $4 \%$ annual pre-tax interest rate \\
\hline - Energy consumption share & $b$ & 0.0084 & Matches steady-state targets \\
\hline \multicolumn{4}{|l|}{ Technology } \\
\hline - Share of physical capital in production & $\beta$ & $1 / 3$ & De La Croix and Michel $(2002)$ \\
\hline - Productivity parameter & $A$ & 0.72 & matches steady-state targets \\
\hline - Share of energy in industrial production & $a$ & 0.017 & Calculated from the U.S. EIA State Energy Data \\
\hline - Elasticity of substitution between energy and non-energy in production & $\sigma_{\mathrm{KL}, \mathrm{E}}$ & 0.5470 & Van der Werf 2008 \\
\hline - Elasticity of substitution between physical capital and labor in production & $\sigma_{\mathrm{K}, \mathrm{L}}$ & 0.3191 & Van der Wert (2008) \\
\hline \multicolumn{4}{|l|}{ Energy } \\
\hline - Energy efficiency in the residential sector & $\varepsilon_{r}$ & 0.65 & ACEEE 2016 \\
\hline - Energy efficiency in the industrial sector & $\varepsilon_{f}$ & 0.7 & ACEEE 2016 \\
\hline - Unitary price of residential energy services & $p_{r}$ & 23.30 & U.S. EIA State Energy Data \\
\hline - Unitary price of firm energy services & $p_{f}$ & 10.67 & U.S. EIA State Energy Data \\
\hline
\end{tabular}

\subsection{Main results}

The transitional impact of an energy efficiency improvement in the industrial sector $\left(\varepsilon_{f}\right)$ is shown in Figure 1(a), The one of an energy efficiency improvement in the residential sector $\left(\varepsilon_{r}\right)$ depicted in Figure 1(b),

Figures 1(a) and 1(b) report in the three first quadrants the deviations from the steady-state values of energy consumption, respectively in industrial sector $\left(E_{f}\right)$, in residential sector $\left(E_{r}\right)$, and in the economy $(E)$-expressed as a \% change-. They also report in the three first quadrants the change induced by a $1 \%$ increase in, both, residential energy efficiency $\left(\varepsilon_{r}\right)$ and production energy efficiency 
$\left(\varepsilon_{f}\right)$. The forth quadrant shows the rebound effect in short- and long-term ${ }^{6}$

In our benchmark case, a 1\% increase in energy efficiency in industrial sector has a large negative impact on residential energy consumption in $\%$ and on wide-economy energy consumption compared to the impact of the same change on the industrial energy consumption. In the short-term the negative impacts are small and rise during the transition which operates very quickly (around one and a half year). Our numerical simulation also highlights that the rebound effect evolves from an initial shortterm value close to unity to a long-term value around 0.3 . This means that in the very short-term, the $1 \%$ increase in industrial energy efficiency induce a high partial rebound effect which vanishes to a small partial rebound effect in the long-term.

Regarding impacts of variations in the residential energy efficiency, i.e. Figure 1(b), results show that $1 \%$ increase in residential energy efficiency reduces energy consumption in, both, the short- and long-term, where the negative short-term impact is lower than the long-term one. The short-term rebound effect on the increase in residential energy efficiency is almost full.

\footnotetext{
${ }^{6}$ The rebound effect is computed as $1+\frac{\Delta E}{E} / \frac{\Delta \varepsilon_{i}}{\varepsilon_{i}}$ with $i=r, f$.
} 


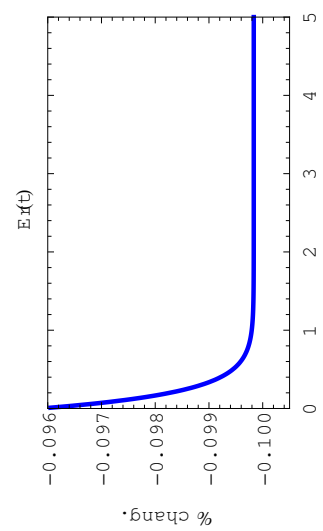

Биеч० \%

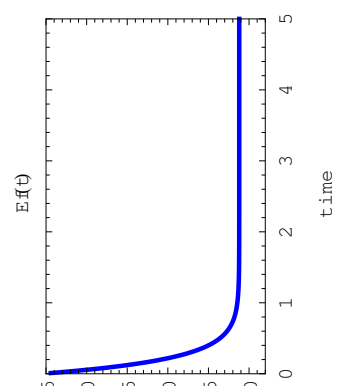

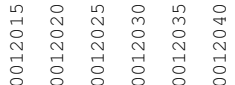

i i i $i$ i

- Биечอ \%

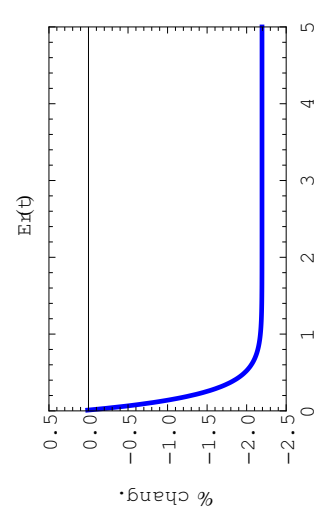

-Биечว \%

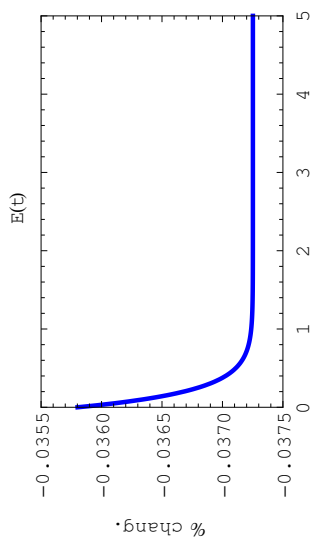

$\hat{a}$

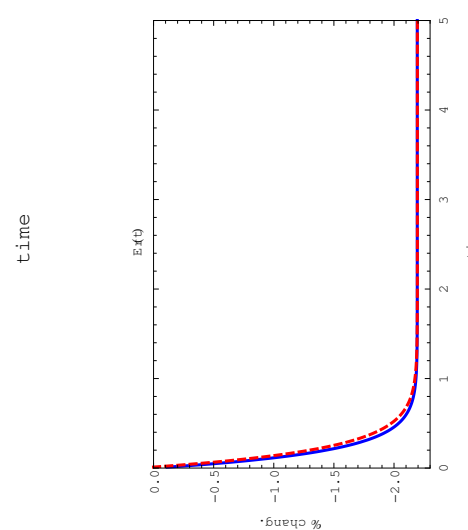

త

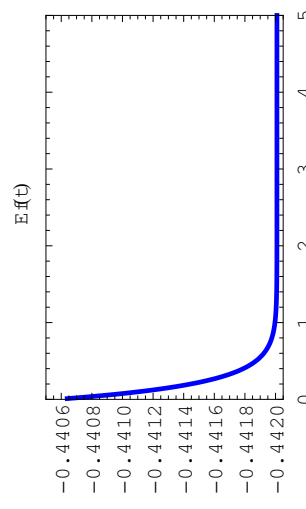

- Биечо \%
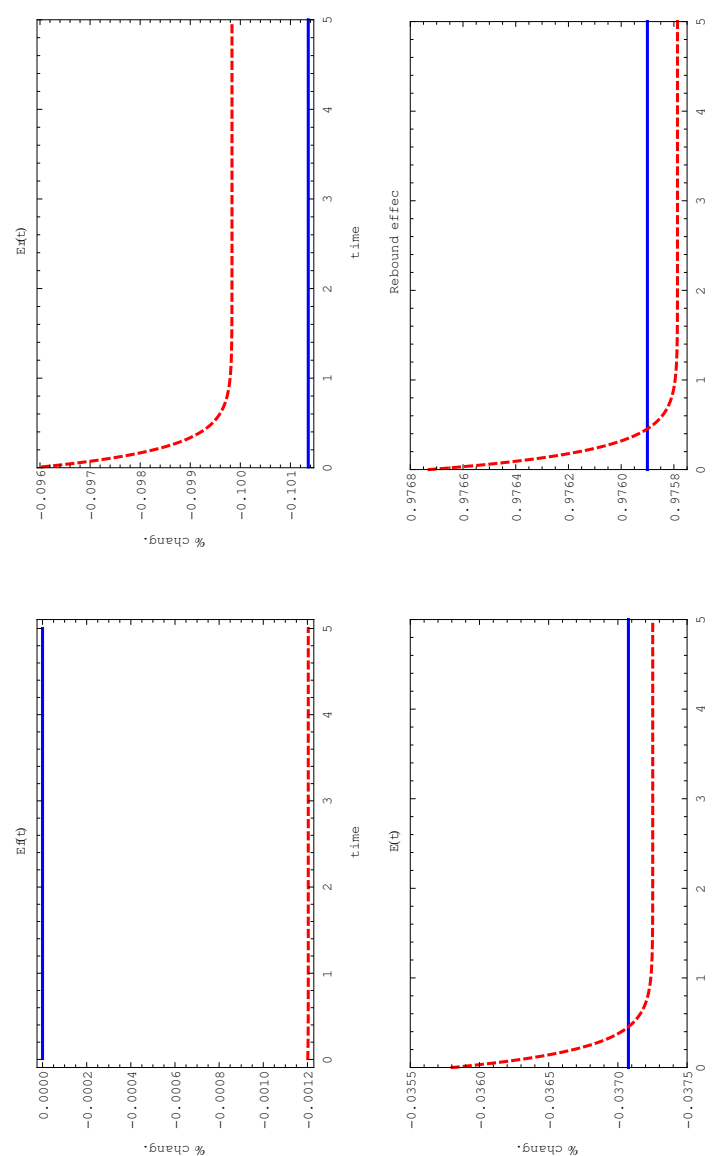

ซ
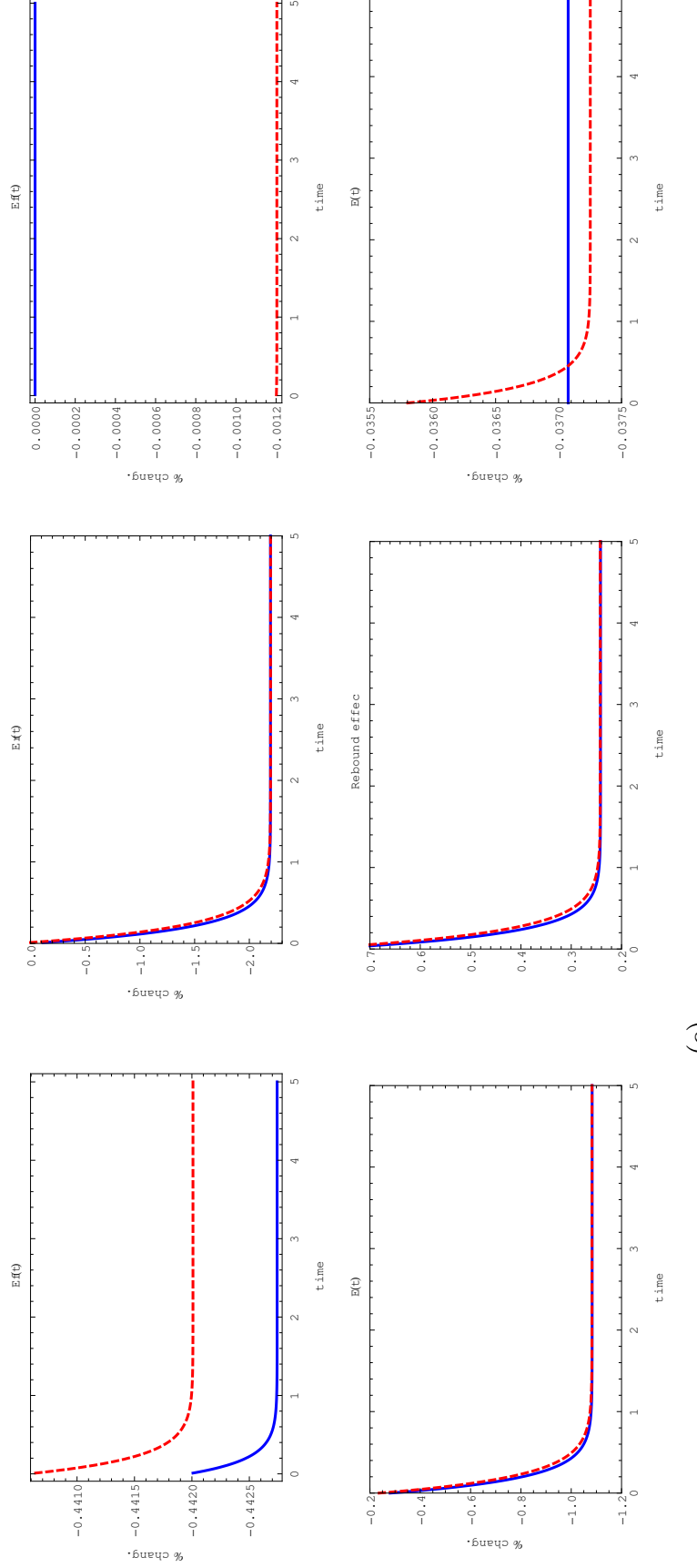
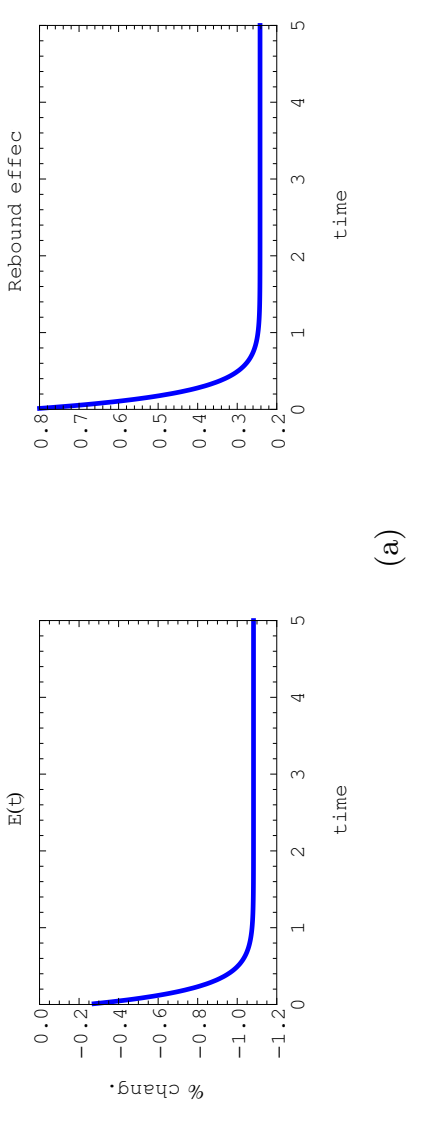

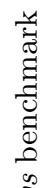


Figures 1(c) and 1(d) respectively show the transitional effects of energy efficiency improvements when labor is exogenous and endogenous. They corroborate the statement of Proposition 1 regarding the short-term steady-state equilibrium: endogenous labor supply generates energy efficiency spillover effects going from the residential sector $\left(\varepsilon_{r}\right)$ into the industrial sector in which the energy consumption increases $\left(E_{f}\right)$. In particular, Figure $1(\mathrm{~d})$ indicates that not taking into account endogenous labor, that is abstracting from intersectoral spillovers, would lead to underestimate the impact of a $1 \%$ increase in residential energy efficiency on industrial energy consumption. It also would lead to overestimate the short- and long-term reduction in residential energy consumption after an energy efficiency improvement. It would also induce an overestimation (respectively underestimation) of the reduction in total energy consumption in the short-term (respectively in the long-term). Finally, it would lead to underestimate (respectively overestimate) the short-term rebound effect in the residential sector (respectively long-term).

In Appendix B, we analyse the robustness of our results. We compute deviations from the steadystate values of energy consumption in residential $\left(E_{r}\right)$ and industrial sectors $\left(E_{f}\right)$ as well as in the economy $(E)$. By the same, we also compute deviations from key parameter values after $1 \%$ increase in, both, residential $\left(\varepsilon_{r}\right)$ and industry energy efficiency $\left(\varepsilon_{f}\right)$. Appendix B shows that the sign of the impacts found in our benchmark case remain the same for all of our alternative parameter values. This means that our benchmark results are reliable. Furthermore, they also show that the magnitude of the effects we highlight could be greater with other choice of parameter values meaning than according to peculiar national situation, the misestimates we highlighted could be larger.

\subsection{The influence of the elasticity of substitution between capital/labor and en- ergy}

Theoretical and empirical literature emphasized how the magnitude and the size of the rebound effect vary depending on the nested structure and the value of the elasticity of substitution in the industrial sector (Saunders (1992), Allan et al. (2007), Van der Werf (2008), Hanley et al. (2009)).

In this section we aim to study the impact of varying the value of elasticity of substitution between capital/labor and energy $\sigma_{\mathrm{KL}, \mathrm{E}}$. In particular, we investigate how the the transitional dynamic found in the benchmark case varies depending on different value of this elasticity of substitution. By referring to Hassler et al. (2012), we investigate two alternative values: $\sigma_{\mathrm{KL}, \mathrm{E}}=0.8$ and $\sigma_{\mathrm{KL}, \mathrm{E}}=0.02$.

Figures 2(a) to 2(d) depict comparative dynamics with respect to the benchmark case plotted as red dashed curves. The main feature is that the transitional effects of energy efficiency improvements are not affected similarly by a high and low elasticity of substitution between energy and non-energy inputs. Effects depends on the location of EEIs: in the residential or in the industrial sector. The influence of energy efficiency improvement in the residential sector $\left(\Delta^{+} \varepsilon_{r}\right)$ on energy consumption is dimly influenced by the level of the elasticity of substitution between energy and non-energy inputs but the spillover effect on industrial energy consumption is amplified when the elasticity of substitution between energy and non-energy inputs is high. The influence of energy efficiency improvement in the industrial sector $\left(\Delta^{+} \varepsilon_{f}\right)$ with high elasticity of substitution between energy and non-energy inputs is reduced in terms of decrease in energy consumption in the industrial sector but amplified in terms of decrease in energy consumption in residential sector and in the economy. High elasticity of substitution between between energy and non-energy inputs in production leads to a negative rebound effect, that is a super-conservation situation (Saunders, 2008). 


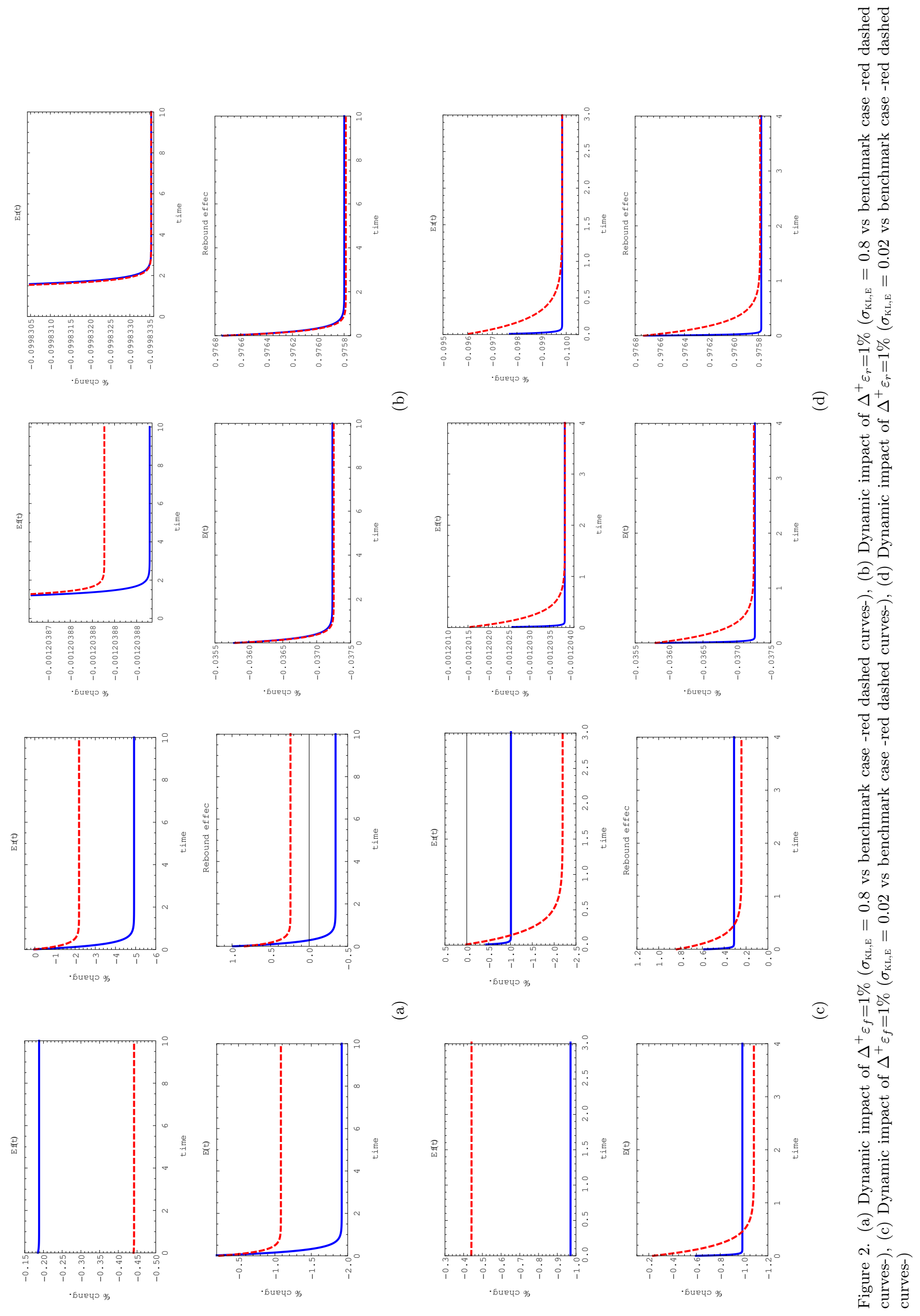




\subsection{The influence of the elasticity of substitution between capital and labor}

While recent empirical studies found a value of physical capital/labor elasticity lower than unity especially for the U.S..$^{7}$ several empirical articles estimating a nested production function with energy found a unitary elasticity of substitution $\sigma_{\mathrm{K}, \mathrm{L}}$. We aim to study how the value of $\sigma_{\mathrm{K}, \mathrm{L}}$ influences the impacts of an energy efficiency variation on energy consumption and rebound effect.

Figures 3(a) and 3(b) show the transitional and long-term effects when $\sigma_{K, L}=1$, a conventional value, and $\sigma_{\mathrm{K}, \mathrm{L}}=0.87$, the upper-bound value of the estimations by Knoblach and Stockl (2020), compared with the benchmark case where $\sigma_{K, L}=0.3191$. These figures highlight two results. First, the higher elasticity of substitution between physical capital and labor rises the time of transition of the economy towards the steady-state, meaning that in the presence of physical capital in production with an elasticity of substitution with respect to labor at the top range of the estimations, the transitional effects of energy efficiency improvements matters. Second, the magnitude of effects of an energy efficiency improvement are quite different with respect to the benchmark case when the elasticity is high. In this context, we observe opposite effects in the short-term with respect to long-term in the case of industrial energy efficiency improvement. In particular, the Figure $3(\mathrm{a})$ shows that a $1 \%$ energy efficiency improvement in the industrial sector leads to a lower decrease in industrial energy consumption and even an increase in residential energy consumption, generating a rebound effect higher than unity in the very short-term and negative in the long-term.

Figure 3(b) shows that differences are less important in the case of a $1 \%$ energy efficiency improvement in the residential sector (with respect to a $1 \%$ energy efficiency improvement in the industrial sector) but that short- and long-term effects have the same sign whatever the targeted sector. They also show that the impact of energy efficiency improvement on the industrial energy consumption is quite different according to the value of $\sigma_{K, L}$. This can be explained by the fact the energy efficiency improvement in the residential sector spillovers towards the industrial sector through the endogenous labor channel (Proposition 1) leading to a lower decrease in overall energy consumption and a higher rebound effect in both the short- and long-term. In addition to highlight the key role of elasticity of substitution between physical capital and labor in determining the magnitude and the transitional dynamic of rebound effect, our results also point out the crucial need to take into account the physical capital when modeling the rebound effect. In particular, not taking into account physical capital would lead to overestimate or underestimate the rebound effect both during the transition stage and in the long-term.

Finally, to illustrate how important is the choice of elasticities of substitution, especially the elasticity of substitution between physical capital/labor and energy, $\sigma_{\mathrm{KL}, \mathrm{E}}$, and between physical capital and labor, $\sigma_{\mathrm{K}, \mathrm{L}}$, Figures $3(\mathrm{c})$ and 3(d) compare transitional and long-term effects using estimated values of $\sigma_{\mathrm{KL}, \mathrm{E}}$ and $\sigma_{\mathrm{K}, \mathrm{L}}$ by Van der Werf (2008) (our benchmark case) and by Bosetti et al. (2006) $\left(\sigma_{\mathrm{KL}, \mathrm{E}}=0.4\right.$ and $\left.\sigma_{K, L}=1\right)$. Differences are quite sizable in both the short- and long-term whatever the type of the energy efficiency improvement. With Bosetti et al. (2006)' parameter values, with a lower $\sigma_{\mathrm{KL}, \mathrm{E}}$ and a higher $\sigma_{K, L}$, transitional impacts of EEIs are longer meaning that during a long time impacts of EEIs on residential and industrial energy consumption, as well as, on global energy consumption may be mis-estimated with Van der Werf (2008)' parameters with respect to Bosetti et al. (2006)' parameters. For example, Figure 3(c) shows that with Bosetti et al. (2006) parameter values, a 1\% increase in industrial energy consumption would rise residential energy consumption by more than 1\% during 5-10 years while, with Van der Werf (2008) parameter values, it would decrease residential energy consumption by more than $2 \%$ from the first year. In the same time, both choices of parameter values lead to similar decreases in residential energy consumption in the very long-term. Conversely, with Bosetti et al. (2006) parameter values, this $1 \%$ increase in industrial energy consumption would lead to a rebound effect higher than unity during 1-2 years and to a rebound effect lower than zero after 25 years while, with Van der Werf (2008) parameter values, it would lead quickly to a stable small positive rebound effect.

\footnotetext{
${ }^{7}$ Performing a meta-analysis from 77 studies between 1961 and 2017 for the U.S. economy, Knoblach and Stockl (2020) estimate a long-run meta-elasticity ranging from 0.45 to 0.87 for te aggregate economy. Using the U.S. private sector data for the period 1948-1998, Antrãs (2004) rejects that the U.S. aggregate production function is Cobb-Douglas.
} 
Our simulations highlight that different estimated values for the elasticities of substitution in production may lead to sizable differences in the magnitude and the duration of the rebound effect. They also show that, besides the elasticity of substitution between physical capital/labor and energy $\left(\sigma_{\mathrm{KL}, \mathrm{E}}\right)$ which has been extensively investigated, the elasticity of substitution between physical capital and labor $\sigma_{\mathrm{K}, \mathrm{L}}$ and between energy and non-energy consumption in utility $\sigma_{\mathrm{C}, \mathrm{E}}$ should be carefully estimated when the rebound effect is empirically evaluated. 

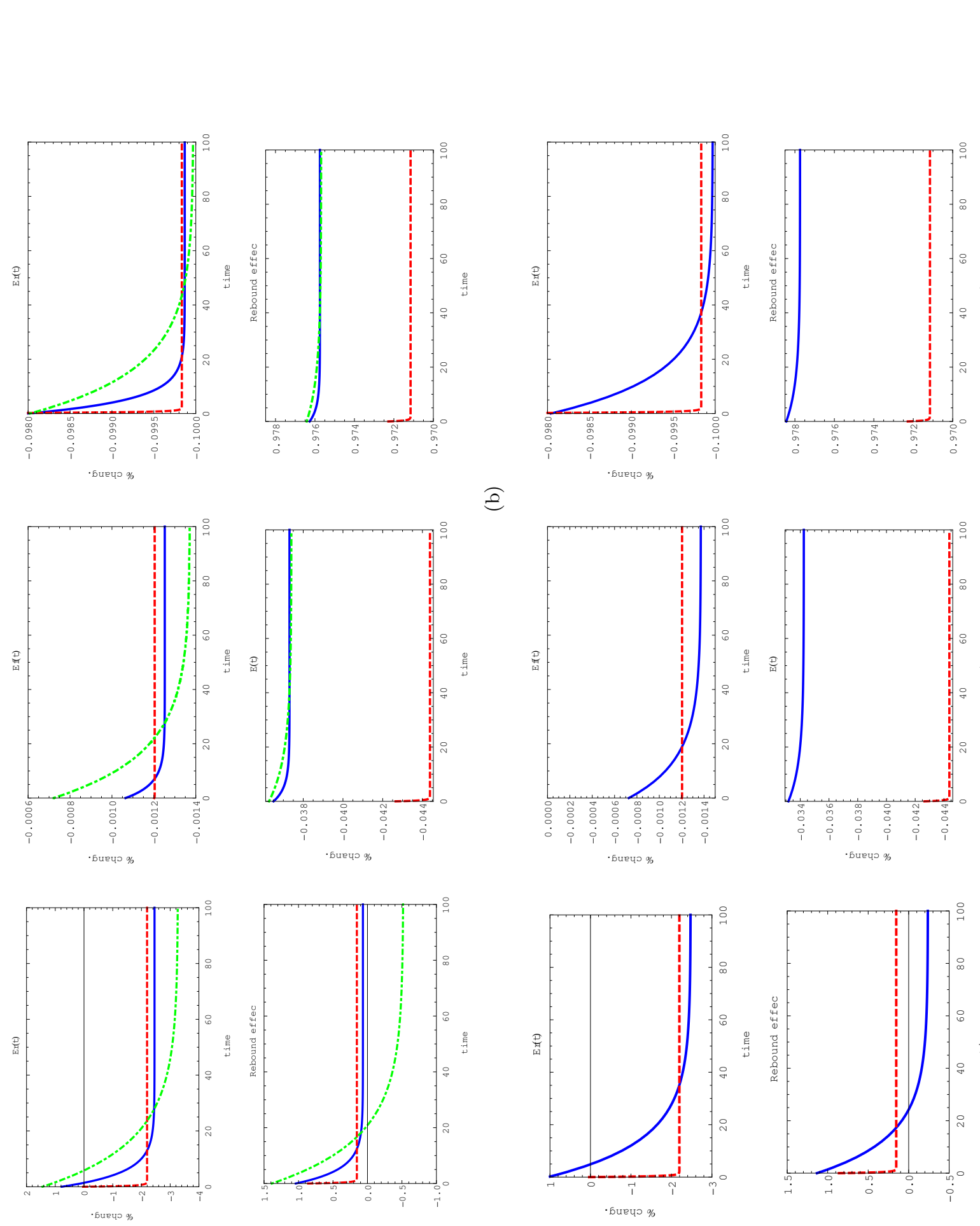

త
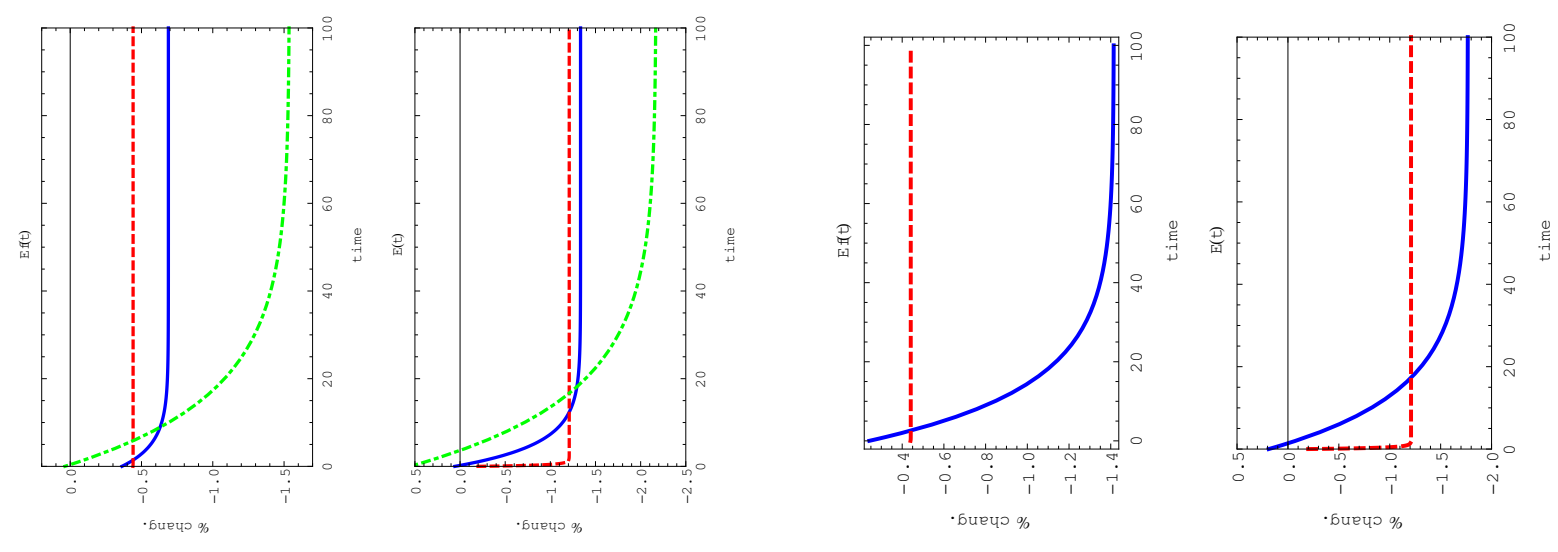

$\stackrel{\infty}{2}$

है

워

के

\begin{tabular}{lll}
4 & 1 \\
\hline & $\infty$ & 0 \\
\hline & 0 \\
\hline & 0 \\
\hline
\end{tabular}

矛芯

:

$e^{2}$

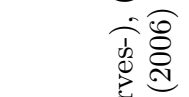

ซ્త

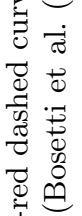

(3)

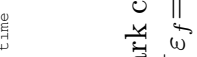

苟㟔

2 音

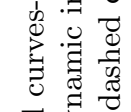

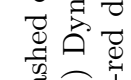

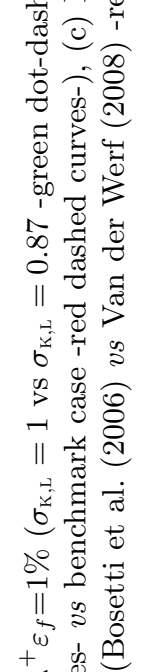

$\checkmark \underbrace{4}$

类

焉焉

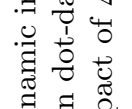

疍芯范

తి

के

호웡 


\section{Conclusion and policy implications}

In this paper, we propose a dynamic general equilibrium model to study effects of energy efficiency improvements and the associated short- and long-term rebound effect. We focus on feedbacks between residential and industrial sectors to identify channels through which an energy efficiency improvement (EEIs) in one sector affect rebound effect in the other sector. We stress the crucial role of the choice of the nesting structure as well as of the size of elasticities of substitution in the production function of firms and in the utility function of households. In the case of the production function, we particularly analyze the impact of varying the elasticity of substitution between capital/labor and energy and the elasticity of substitution between capital and labor.

Our main contributions are twofold. First, we demonstrate that energy efficiency improvements in one sector may spillover into the other sector to directly affect its energy consumption, thus, the magnitude of associated rebound effect. It also affects the magnitude of the rebound effect in the sector in which the energy efficiency improvement occurred as well as the wide-economy energy consumption. We demonstrate that the spillovers of EEIs in residental sector into industrial sector go through the labor supply channel and that the spillovers of EEIs in industrial sector into residential sector go through the income channel. Numerical simulations show that such a spillovers affect the rebound effect in both the short- and long- term.

Our second main contribution is related to the role played by the elasticities of substitution in the size and duration of rebound effects, whatever the sector in which EEIs are implemented. Our theoretical model demonstrates that the size and the sign of inter-sectorial spillovers depends on the values of the elasticity of substitution between physical capital and labor in production and of the elasticity of substitution between non-energy and energy consumptions in utility. This result is in sharp contrast with studies who emphasized the role of the elasticity of substitution between physical capital/labor and energy. It also highlights the importance to introduce physical capital in the general equilibrium analysis of the rebound effect. Finally, it states the role played by household preferences in terms of energy, even in the consequences of EEIs in industrial sector. Our numerical simulations emphasize the importance of the values chosen for these elasticities of substitution. A comparative analysis of the short- and long- term effect of EEIs depending on whether we select values estimated by Van der Werf (2008) or values estimated by Bosetti et al. (2006) reveals sizable differences in the size and the duration of the rebound effect. This result calls for systematic evaluations of the elasticities of substitution between physical capital and labor in production and between non-energy and energy consumption in utility, in a similar way that it has be done for the elasticity of substitution between non-energy and energy inputs in production.

A possible extension of our model is to take into account the sector of energy production and multiple consumption goods rather than one. It would allow for a more comprehensive analysis of sectoral interactions, thus, a more detailed study of the rebound effect through a tiny decomposition of the general equilibrium response to an energy efficiency improvement. An another interesting extension is to investigate alternative utility functions to understand how the shape of the utility function and the way non-energy consumption and energy consumption enters it would affect our results.

In policy terms, in the context of recurrent worldwide debates about energy, environmental, and climate policies, our results suggest that the type of energy efficiency policies to be implemented should take into account sectoral feedbacks, in particular, the potential for rebound effect. Academic literature has credibly demonstrated that rebound effect that may appear in one or several sectors can offset benefits for the economy from an energy efficiency policies. For this reason, the policy inaction on the rebound effect was for a while criticized. The definition and implementation of energy efficiency policies in particular, and of environmental and climate policies in general, should be associated with rebound mitigation strategies. This recall for reconsidering the place of the rebound effect issue in the policy agenda as well as policy pathways that can be effective in offsetting negative impacts of rebound effect. 


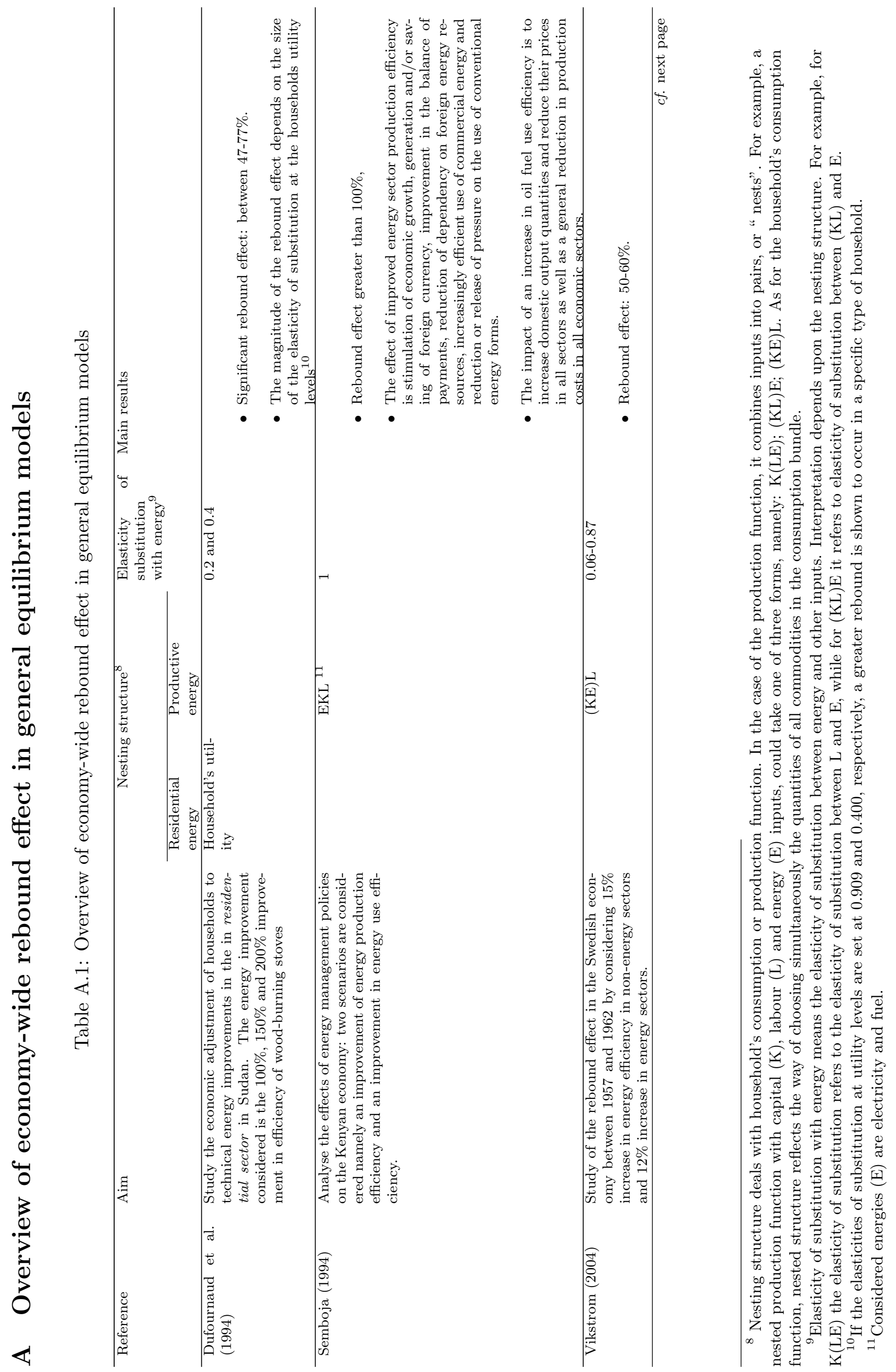




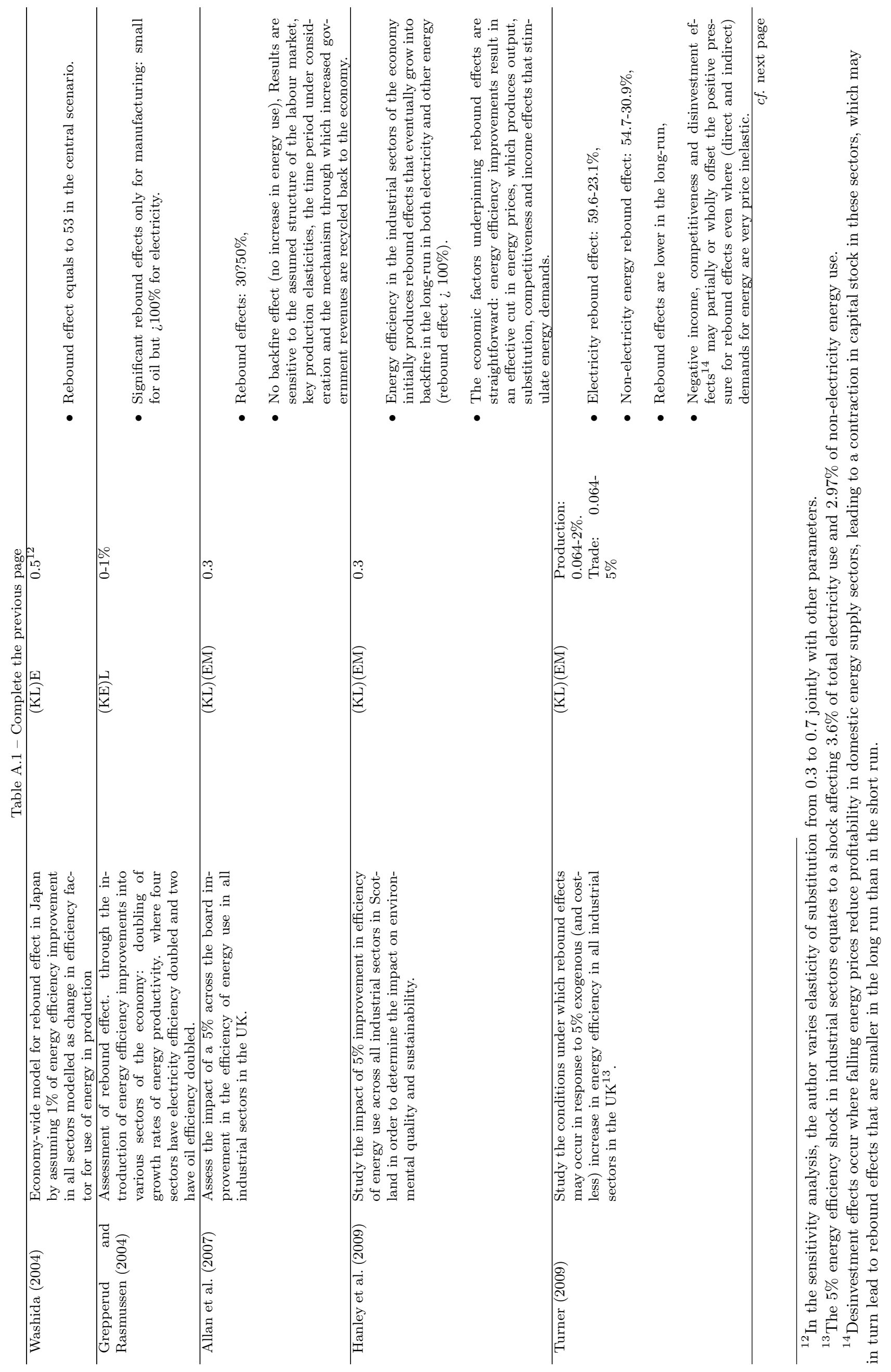




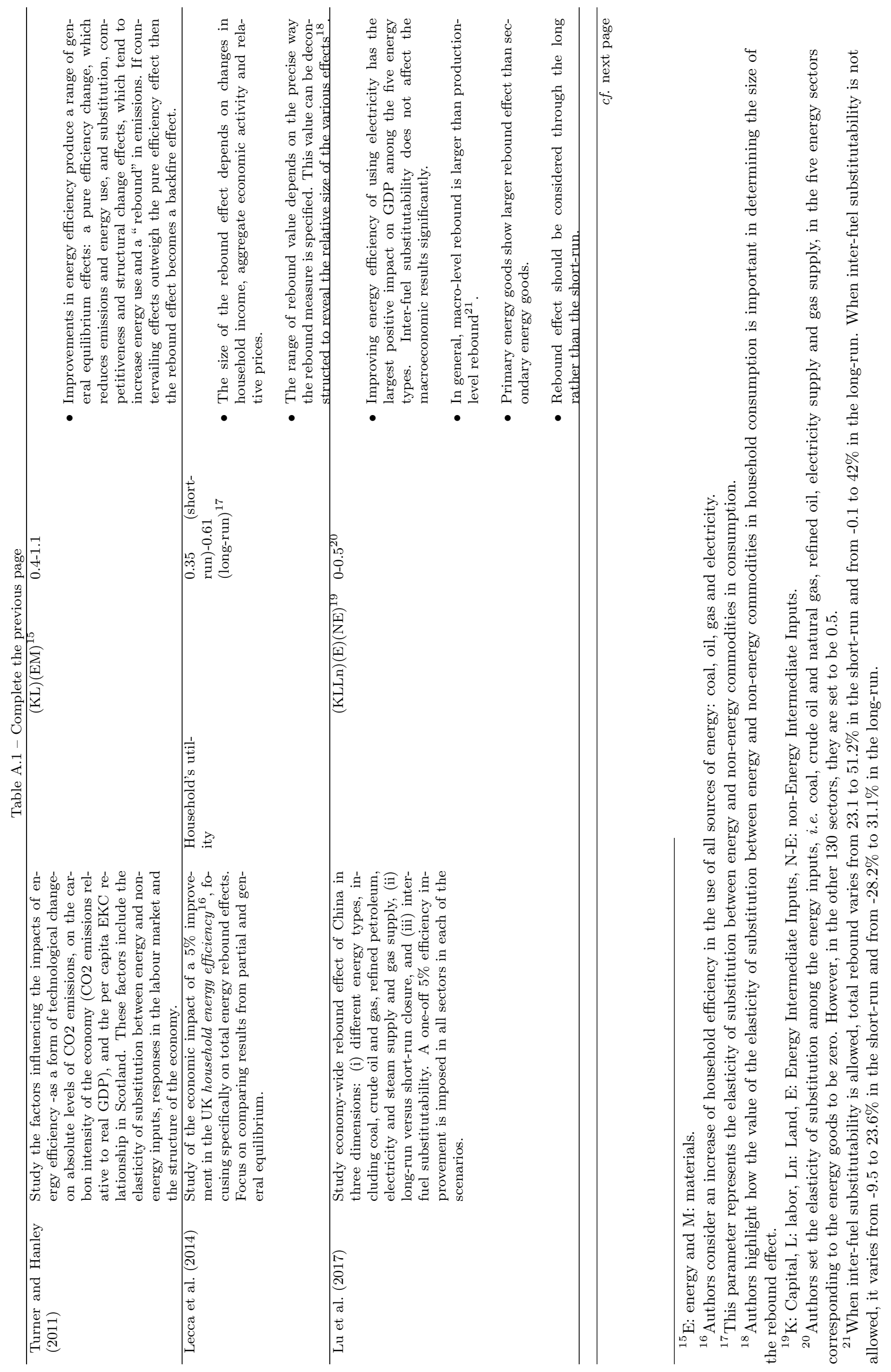




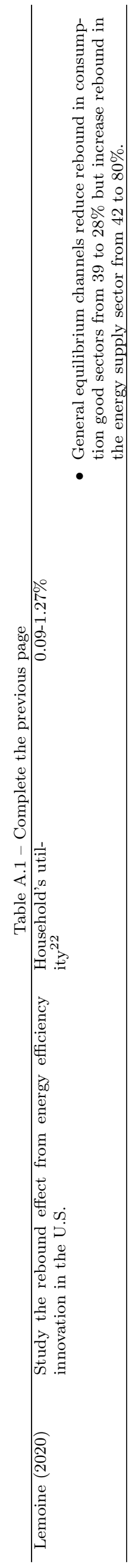



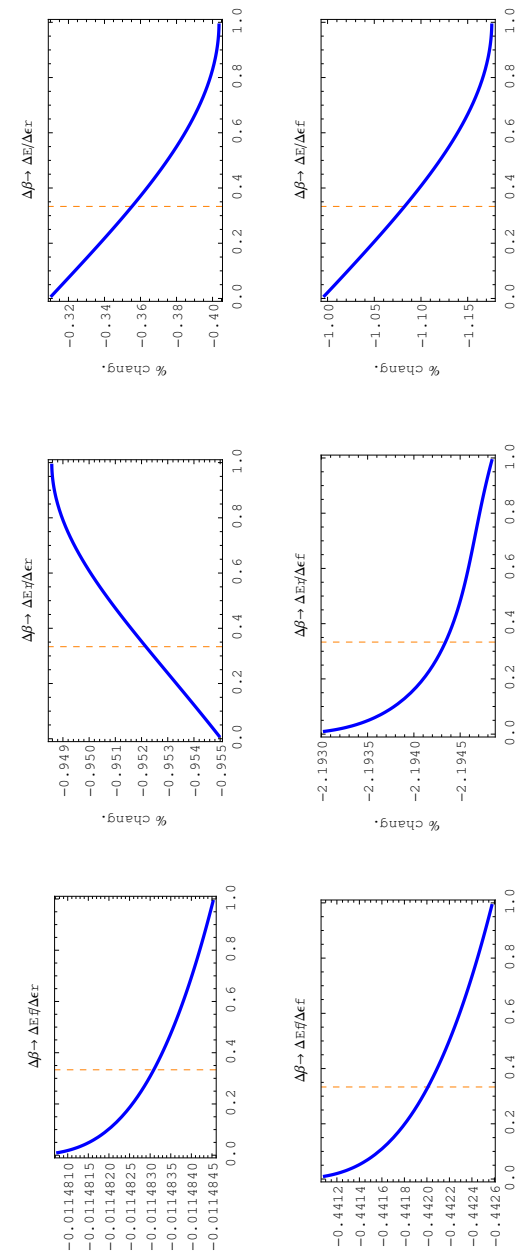

- Биечг \%
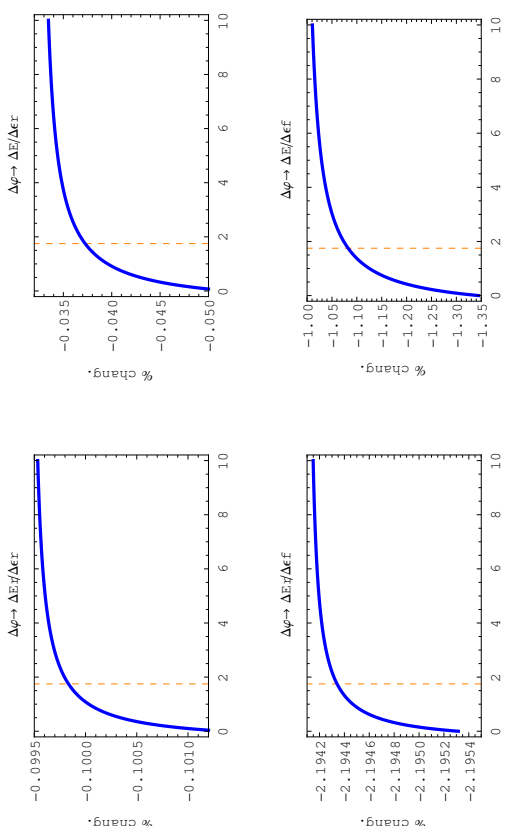

త
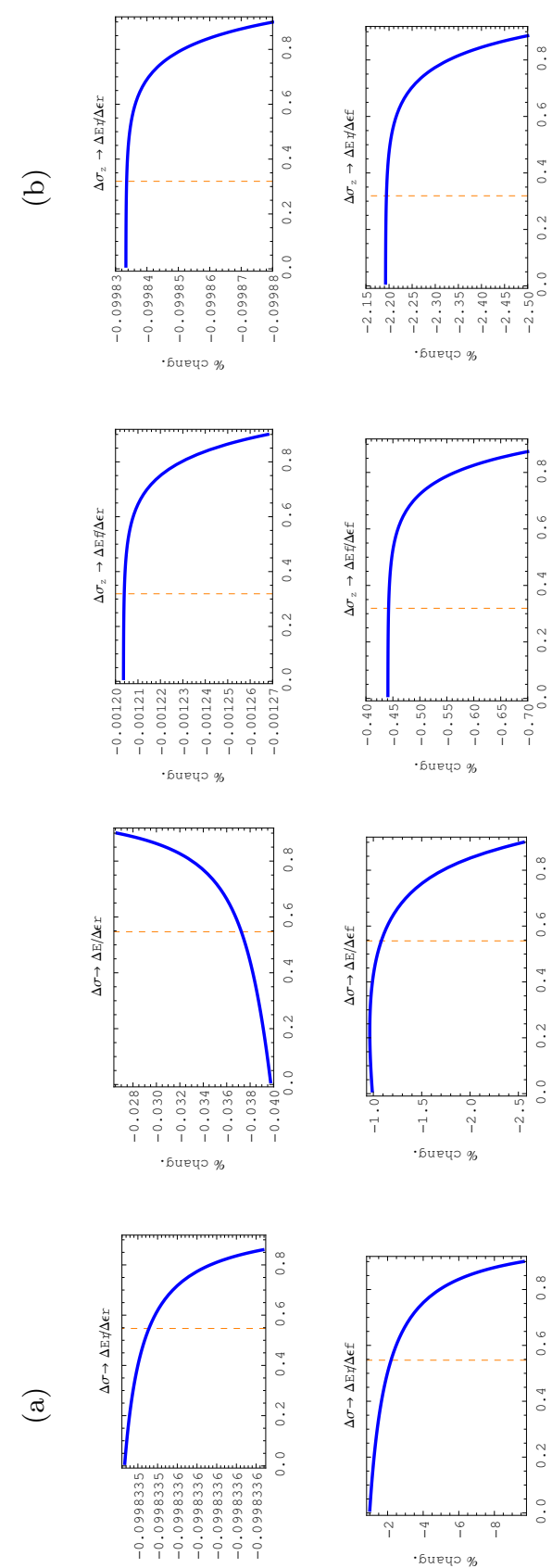

- Биечо \%
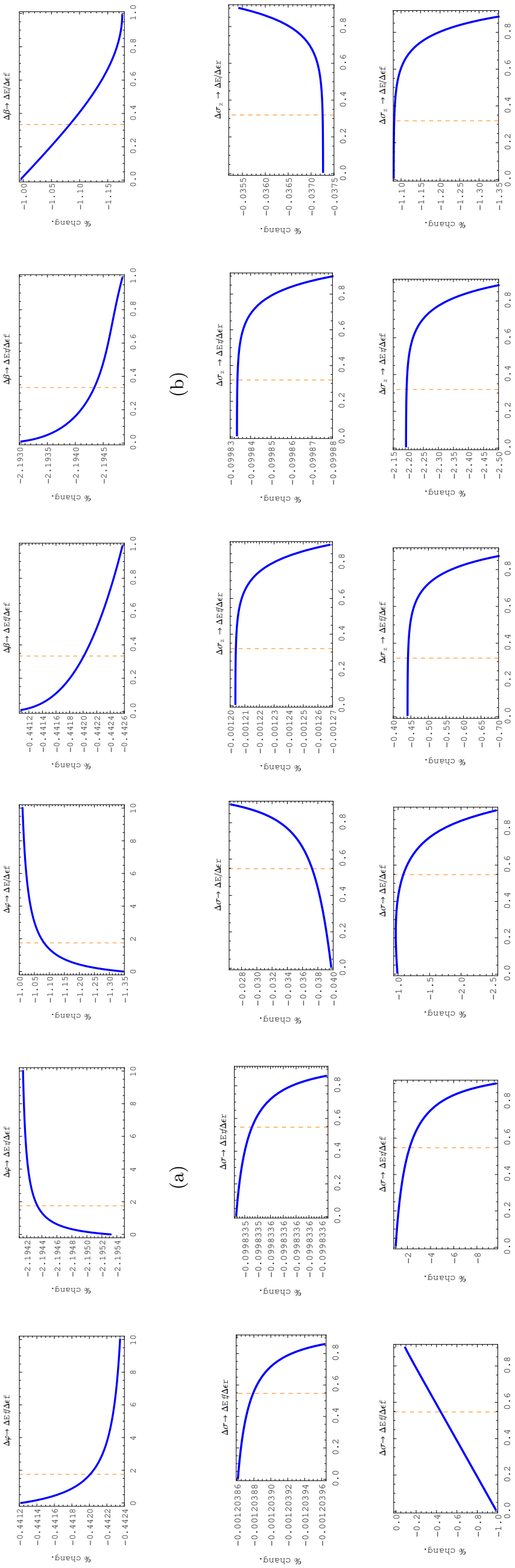

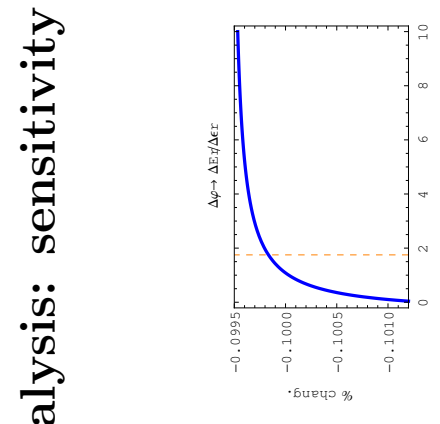

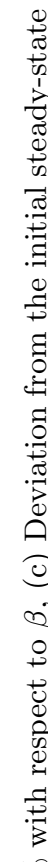

3

要

告

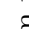

을

.

인

อิ

웅

苋

表荡

营旁

5

营. 宽

(e)

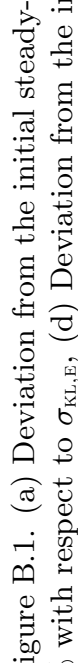

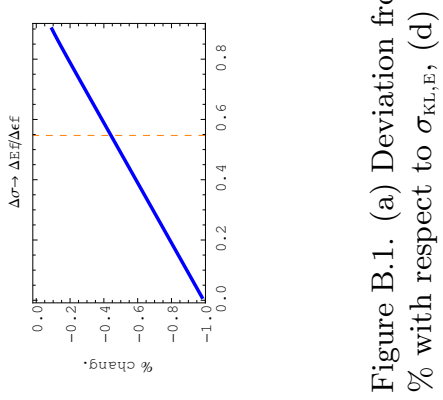



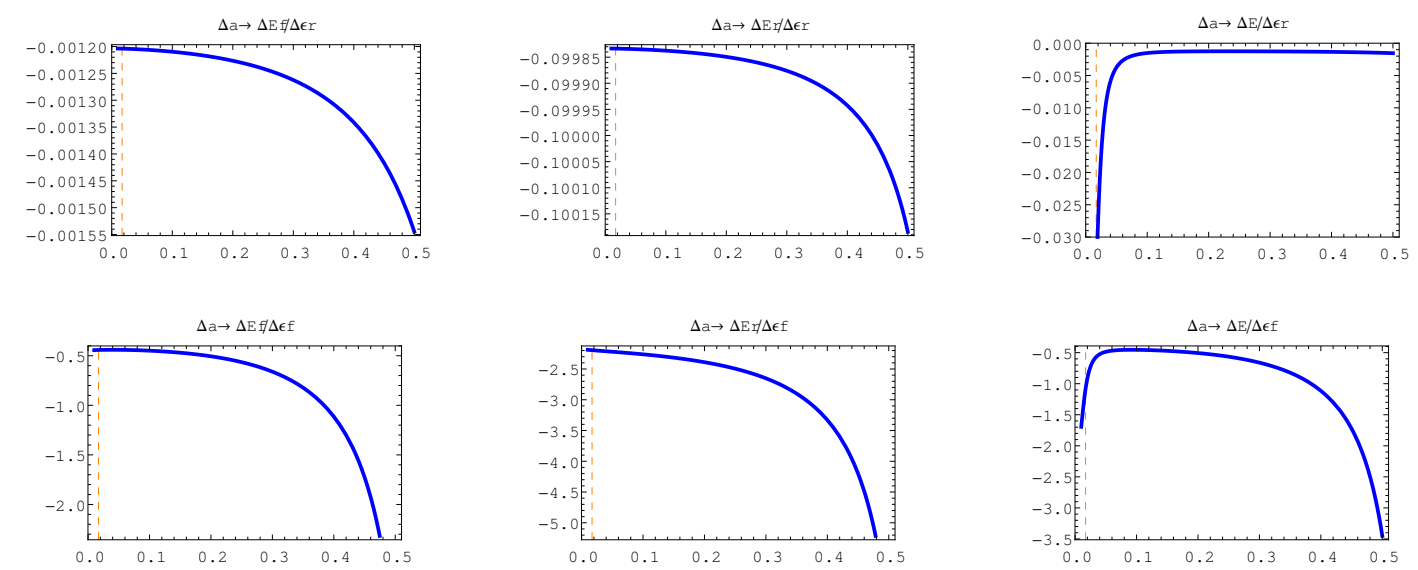

Figure B.2: . Deviation from the initial steady-state $\%$ with respect to $a$ 


\section{References}

Allan, G., M. Gilmartin, P. McGregor, J. Swales, and K. Turner (2009). Energy efficiency and sustainable consumption: The rebound effect, Chapter Modelling the economy-wide rebound effect, pp. 69-98. Palgrave Macmilan.

Allan, G., N. Hanley, P. McGregor, K. Swales, and K. Turner (2007). The impact of increased efficiency in the industrial use of energy: A computable general equilibrium analysis for the United Kingdom. Energy Economics (29), 779-798.

Antrãs, P. (2004). Is the U.S. aggregate production function Cobb-Douglas? New estimates of the elasticity of substitution. The B.E. Journal of Macroeconomics $4(1)$.

Borenstein, S. (2015). A microeconomic framework for evaluating energy efficiency rebound and some implications. Energy Journal 36, 1-21.

Bosetti, V., C. Carraro, M. Galeotti, E. Messetti, and M. Tavoni (2006). A world induced technical change hybrid model. The Energy Journal 27, 13-37.

Brookes, L. G. (1990). The greenhouse effect: Fallacies in the energy efficiency solution. Energy Policy 3, 199-201.

De La Croix, D. and P. Michel (2002). A Theory of Economic Growth : dynamics and policy in overlapping generations. Cambridge University Press.

de Miguel, C. and B. Manzano (2011). Green tax reforms and habits. Resource and Energy Economics 33(1), 231-246.

Dimitropoulos, J. (2007). Energy productivity improvements and the rebound effect: An overview of the state of knowledge. Energy policy 35, 6354-6363.

Druckman, A., M. Chitnis, S. Sorrell, and T. Jackson (2011). Missing carbon reductions? Exploring rebound and backfire effects in UK households. Energy policy 39(26), 3572-3581.

Dubin, J. A., A. K. Miedema, and R. V. Chandran (1986). Price effects of energy-efficient technologies - A study of residential demand for heating and cooling. Rand Journal of Economics 17(3), 310-325.

Dufournaud, C., J. T. Quinn, and J. Harrington (1994). An applied general equilibrium (AGE) analysis of a policy designed to reduce the household consumption of wood in the Sudan. Resource and Energy Economics 16, 67-90.

Eto, J., S. Kito, L. Shown, and R. Sonnenblick (1995). Where did the money go? The cost and performance of the largest commercial DSM programs. The Energy Journal 21 (2), 23-50.

Freire-Gonzalez, J. (2011). Methods to empirically estimate direct and indirect rebound effect of energy-saving technological changes in households. Ecological Modelling 223(1), 32-40.

Frondel, M., J. Horbach, and K. Rennings (2008). What triggers environmental management and innovation? Empirical evidence for Germany. Ecological Economics 66(1), 153-160.

Greene, D. L., J. R. Khan, and R. C. Gibson (1999). Fuel economy rebound effect for us household vehicles. Energy Journal 20(3), 1-31.

Greening, L. A., D. L. Greene, and C. Difiglio (2000). Energy efficiency and consumption. The rebound effect. A survey. Energy Policy 28(6-7), 389-401.

Grepperud, S. and I. Rasmussen (2004). A general equilibrium assessment of rebound effects. Energy economics 26(2), 282-261.

Hanley, N., P. G. McGregor, J. K. Swales, and K. Turner (2009). Do increases in energy efficiency improve environmental quality and sustainability? Ecological Economics 68, 692-709. 
Hassler, J., P. Krusell, and C. Olovsson (2012, October). Energy-saving technical change. NBER Working Paper 18456, National Bureau of Economic Research, Cambridge, M.A.

Jevons, W. S. (1865). The coal question: An inquiry concerning the progress of the nation, and the probable exhaustion of our coal mines. MacMillan, London.

Khazzoom, J. D. (1980). Economic implications of mandated efficiency in standards for household appliances. Energy Journal 1(4), 21-40.

Khazzoom, J. D. (1987). Energy savings from the adoption of more efficient appliance. The Energy Journal 8(4), 85-89.

Klein, Y. L. (1987). Residential energy conservation choices of poor households during a period of rising energy prices. Resources and Energy 9(4), 363-378.

Klein, Y. L. (1988). An econometric model of the joint production and consumption of residential space heat. Southern Economic Journal 55(2), 351-359.

Knoblach, M. and F. Stockl (2020). What determines the elasticity of substitution between capital and labor? A literature review. Journal of Economic Surveys, https://doi.org/10.1111/joes.12366.

Lecca, P., P. G. McGregor, J. Kim Swales, and K. Turner (2014). The added value from a general equilibrium analysis of increased efficiency in household energy use. Ecological Economics 100, $51-62$.

Lemoine, D. (2020). General equilibrium rebound from energy efficiency innovation. European Economic Review 125, 103431.

Lu, Y., Y. Liu, and M. Zhou (2017). Rebound effect of improved energy efficiency for different energy types: A general equilibrium analysis for china. Energy economics 62, 248-256.

Nadel, S. (1993). The take-back effect: fact or fiction. U933, ACEEE.

Orea, L., M. Llorca, and M. Filippini (2015). A new approach to measuring the rebound effect associated to energy efficiency improvements: An application to the U.S. residential energy demand. Energy Economics 49, 599-609.

Prescott, E. C. (2004). Why do Americans work so much more than europeans? Federal Reserve Bank of Minneapolis Quarterly Review 28(1), 2-13.

Saunders, H. D. (1992). The Khazzoom-Brookes postulate and neoclassical growth. The Energy Journal 13, 131-148.

Saunders, H. D. (2008). Fuel conserving (and using) production functions. Energy Economics 30(5), $2184-2235$.

Schwarz, P. M. and T. N. Taylor (1995). Cold hands, warm hearth? Climate, net takeback, and household comfort. Energy Journal 16(1), 41-54.

Semboja, H. H. H. (1994). The effect of an increase in energy efficiency on the Kenyan economy. Energy policy March, 217-225.

Sorrell, S. and J. Dimitropoulos (2008). The rebound effect: Microeconomic definitions, limitations and extensions. Ecological Economics 65(636-649).

Sorrell, S., J. Dimitropoulos, and M. Sommerville (2009). Empirical estimates of the direct rebound effect: A review. Energy policy 37, 1356-1371.

Thomas, B. A. and I. L. Azevedo (2013a). Estimating direct and indirect rebound effects for U.S. households with input-output analysis part 1: Theoretical framework. Ecological Economics (a)86, 199-210. 
Thomas, B. A. and I. L. Azevedo (2013b). Estimating direct and indirect rebound effects for U.S. households with input-output analysis part 2: Simulation. Ecological Economics (b)86(188-198).

Turner, K. (2009). Negative rebound and disinvestment effects in response to an improvement in energy efficiency in the UK economy. Energy economics 31, 648-666.

Turner, K. (2013). Rebound effects from increased energy efficiency: A time to pause and reflect. The Energy Journal 34, 25-42.

Turner, K. and N. Hanley (2011). Energy efficiency, rebound effects and the Environmental Kuznets Curve. Energy economics 33(5), 709-720.

Turnovsky, S. J. and C. Garcia-Penalosa (2008). Distributional dynamics in a neoclassical growth model: The role of elastic labor supply. Journal of Economic Dynamics and Control 32(5), 13991431.

Van den, Bergh, J. C. (2011). Energy conservation more effective with rebound policy. Environment and Resource Economics 48, 43-58.

Van der Werf, E. (2008). Production functions for climate policy modeling: An empirical analysis. Energy economics 30(6), 2964-2979.

Vikstrom, P. (2004). Energy efficiency and energy demand: A historical CGE investigation on the rebound effect in the Swedish economy 1957. Dept. of Economic History. Umea University.

Washida, T. (2004). Economy-wide model of rebound effect for environmental efficiency. International Workshop on Sustainable Consumption, University of Leeds.

West, S. E. (2004). Distributional effects of alternative vehicle pollution control policies. Journal of Public Economics 88(735-757). 


\section{NOTE DI LAVORO DELLA FONDAZIONE ENI ENRICO MATTEI \\ Fondazione Eni Enrico Mattei Working Paper Series}

Our Working Papers are available on the Internet at the following addresses:

http://www.feem.it/getpage.aspx?id=73\&sez=Publications\&padre=20\&tab=1

\section{NOTE DI LAVORO PUBLISHED IN 2020}

1. 2020, FACTS Series, Alessandra Celani de Macedo, Nicola Cantore, Laura Barbier, Marco Matteini, Giorgia Pasqualetto, The Impact of Industrial Energy Efficiency on Economic and Social Indicators

2. 2020, 2030 Agenda Series, Nicola Comincioli, Sergio Vergalli, Effects of Carbon Tax on Electricity Price Volatility: Empirical Evidences from the Australian Market

3. 2020, 2030 Agenda Series, Marco Buso, Cesare Dosi, Michele Moretto, Do Exit Options Increase the Value-For-Money of Public-Private Partnerships?

4. 2020, FACTS Series, Ricardo Nieva, A Tragic Solution to the Collective Action Problem: Implications for Corruption, Conflict and Inequality

5. 2020, FACTS Series, Charles Fang Chin Cheng, Nicola Cantore, The Inclusive and Sustainable Development Index: a Data Envelopment Analysis Approach

6. 2020, 2030 Agenda Series, FACTS Series, Coker Eric, Cavalli Laura, Fabrizi Enrico, Guastella Gianni, Lippo Enrico, Parisi Maria Laura, Pontarollo Nicola, Rizzati Massimiliano, Varacca Alessandro, Vergalli Sergio, The Effects of Air Pollution on COVID-19 Related Mortality in Northern Italy

7. 2020, 2030 Agenda Series, Laura Cavalli, Giulia Lizzi, Port of the future : Addressing Efficiency and Sustainability at the Port of Livorno with $5 \mathrm{G}$

8. 2020, FACTS Series, Federica Cappelli, Gianni Guastella, Stefano Pareglio, Institutional Fragmentation and Urbanisation in the EU Cities

9. 2020, FEP Series, Giacomo Falchetta, Nicolò Stevanato, Magda Moner-Girona, Davide Mazzoni, Emanuela Colombo and Manfred Hafner, M-LED: Multi-sectoral Latent Electricity Demand Assessment for Energy Access Planning

10. 2020, Local Projects Series, Marcella De Filippo, Annalisa Percoco, Angela Voce, Covid-19 e didattica a distanza.Il caso Basilicata, una regione a rischio digital divide

11. 2020, 2030 Agenda, Laura Cavalli, Sandro Sanna, Mia Alibegovic, Filippo Arras, Gianluca Cocco, Luca Farnia, Emanuela Manca, Luisa F. Mulas, Marco Onnis, Sandro Ortu, Ilenia G. Romani, Marta Testa, The Contribution of the European Cohesion Policy to the 2030 Agenda: an Application to the Autonomous Region of Sardinia

12. 2020, FACTS Series, Alexander Golub, Kristina Govorukha, Philip Mayer, Dirk Rübbelke, How does Climate Change Affect the Transition of Power Systems: the Case of Germany

13. 2020, FEP Series, Rossana Scita, Pier Paolo Raimondi and Michel Noussan, Green Hydrogen: the Holy Grail of Decarbonisation? An Analysis of the Technical and Geopolitical Implications of the Future Hydrogen Economy

14. 2020, FACTS Series, Marta Montinaro, Rupayan Pal, Marcella Scimitore, Per Unit and Ad Valorem Royalties in a Patent Licensing Game

15. 2020, 2030 Agenda, S. Quaini, S. Saccani, S. Vergalli, L. Assom, M. Beria, A. Codello, M. Monaco, R. Sabatini, Seasonality Fingerprint on Global Trading of Food-commodities. A Data-mining Approach

16. 2020, 2030 Agenda Series, Nicola Comincioli, Paolo M. Panteghini and Sergio Vergalli, Debt and Transfer Pricing: Implications on Business Tax Policy

17. 2020, FACTS Series, Wolfgang Buchholz, Dirk Rübbelke, Overstraining International Climate Finance: When Conflicts of Objectives Threaten Its Success

18. 2020, 2030 Agenda Series, Nicola Comincioli, Verena Hagspiel, Peter M. Kort, Francesco Menoncin, Raffaele Miniaci and Sergio Vergalli, Mothballing in a Duopoly: Evidence from a (Shale) Oil Market

19. 2020, 2030 Agenda Series, Chiara Castelli and Angela Parenti, Commuting in Europe: An Inter-regional Analysis on its Determinants and Spatial Effects

20. 2020, 2030 Agenda Series, Iwan Bos, Marco A. Marini, Collusion in Quality-Segmented Markets

21. 2020, FACTS Series, Federica Cappelli, Investigating the Origins of Differentiated Vulnerabilities to Climate Change and their Effects on Wellbeing 
22. 2020, 2030 Agenda Series, Antonio Francesco Gravina, Matteo Lanzafame, Nonlinearities and the determinants of inequality: New panel evidence

23. 2020, FACTS Series, Jane da Mosto, Camilla Bertolini, Anil Markandya, Paulo A.L.D. Nunes, Tom Spencers, Arnas Palaimas, Laura Onofri, Rethinking Venice from an Ecosystem Services Perspective

24. 2020, FACTS Series, Maria Rosaria Alfano, Anna Laura Baraldi, Erasmo Papagni, Do Voters Choose Better Politicians than Political Parties? Evidence from a Natural Experiment in Italy

25. 2020, FACTS Series, Vesa-Pekka Parkatti, Olli Tahvonen, Economics of multifunctional forestry in the Sàmi people homeland region

26. 2020, 2030 Agenda Series, Michael Kopel, Marco A. Marini, Mandatory Disclosure of Managerial Contracts in Nonprofit Organizations

27. 2020, 2030 Agenda Series, lan W. R. Martin, Robert S. Pindyck, Welfare Cost of Catastrophes: Lost Consumption and Lost Lives

28. 2020, Future Energy Program Series, Sondes Kahouli, Xavier Pautrel, Residential and industrial energy efficiency improvements: A dynamic general equilibrium analysis of the rebound effect 


\section{Fondazione Eni Enrico Mattei}

Corso Magenta 63, Milano - Italia

Tel. +39 02.520.36934

Fax. +39.02 .520 .36946$

E-mail: letter@feem.it 